\title{
The optical singularities of birefringent dichroic chiral crystals $\dagger$
}

\author{
BY M. V. BERRY AND M. R. DENNIS \\ H. H. Wills Physics Laboratory, Tyndall Avenue, Bristol BS8 1TL, UK
}

Received 7 February 2003; accepted 10 March 2003; published online 27 March 2003

Using a new formalism involving projection from the sphere of directions to the stereographic plane, and associated complex variables, explicit formulae are obtained for the two refractive indices and polarizations in optically anisotropic crystals that are both dichroic (absorbing) and chiral (optically active). This enables three types of polarization singularity to be classified and explored: singular axes, which are degeneracies where the two refractive indices are equal, and which for a transparent non-chiral crystal condense pairwise onto the optic axes; $\mathrm{C}$ points, where the polarization is purely circular (right- or left-handed), with topological index +1 , $+\frac{1}{2}$ or $+\frac{1}{4}$ and whose positions are independent of the chirality; and L lines, where the polarization is purely linear, dividing direction space into regions with rightand left-handedness. A local model captures essential features of the general theory. Interference figures generated by slabs of crystal viewed directly or through a polarizer and/or analyser enable the singularities to be displayed directly.

Keywords: polarization; crystal optics; singularities; degeneracies; stereographic projection

\section{Introduction}

The classical linear wave optics of homogeneous non-magnetic crystals has been studied for several centuries. Its firm basis in Maxwell's equations is well understood (Born 1933; Born \& Wolf 1959; Landau et al. 1984; Nye 1995), and the subject has been comprehensively and authoritatively reviewed (Ramachandran \& Ramaseshan 1961). Nevertheless, the physics and the underlying mathematics can appear complicated, especially in the most general case of crystals that possess natural optical activity (chirality) and dichroism (absorption) in addition to biaxial birefringence. Our purpose here is to develop the theory of crystal optics in a fresh way, which unifies and clarifies the many different phenomena that can occur. To achieve this, we combine a geometrical approach, emphasizing the several types of singularity, with an algebraic formulation that makes calculations easy.

Classical crystal optics provides beautiful illustrations and extensions of the generic polarization singularities of optical fields, in which there is much current interest (Nye 1983a, 1999; Angelsky et al. 2002; Freund et al. 2002; Dennis 2002; Freund 2002). This is part of a wider exploration of optical singularities, for example, phase dislocations (Nye \& Berry 1974; Vasnetsov \& Staliunas 1999; Berry 1998) and caustics

$\dagger$ Dedicated to Professor J. F. Nye, FRS, and Professor S. Ramaseshan on their 80th birthdays.

Proc. R. Soc. Lond. A (2003) 459, 1261-1292

(C) 2003 The Royal Society 
and associated diffraction (Berry \& Upstill 1980; Nye 1999). The singularities are our principal interest: therefore, our focus is not the usual one, in which polarized light is employed to identify crystals, but rather to use crystals as vehicles to identify and explore polarization singularities.

The polarizations of the two plane waves that can propagate in an anisotropic medium are fully described by their electric displacement vectors, which can be written in the form

$$
\boldsymbol{D}(\boldsymbol{s}) \exp \{\mathrm{i} k(n(\boldsymbol{s}) \boldsymbol{s} \cdot \boldsymbol{r}-c t)\} .
$$

Here, the frequency $c k$ and the unit direction $s$ are specified, and the two refractive indices $\boldsymbol{n}(\boldsymbol{s})$ and the associated complex polarization vectors $\boldsymbol{D}(\boldsymbol{s})$ are to be determined. We choose $\boldsymbol{D}$, rather than the electric field $\boldsymbol{E}$, because for plane waves $\boldsymbol{D}$ is always transverse (i.e. $\boldsymbol{D}(\boldsymbol{s}) \cdot \boldsymbol{s}=0$ ), whereas $\boldsymbol{E}$ generally is not. (If we were considering rays rather than waves, that is, the direction of energy flow, then $\boldsymbol{E}$ would be the primary vector (Ramachandran \& Ramaseshan 1961; Born \& Wolf 1959), but we do not pursue this aspect here.) Our emphasis throughout will be on the dependence on direction $s$, though we will not always indicate this explicitly. We will be interested in how the various patterns in $\boldsymbol{s}$-space vary as parameters describing the crystal are changed - that is, how the patterns change with position in 'crystal space' - and will illustrate the theoretical analysis of the patterns with pictures computed numerically. (Since we are considering plane waves, the polarizations are spatially uniform, so the waves do not possess a skeleton of singular lines in space, as in more familiar applications of singular optics (Nye 1999).)

The crystal will be specified by its inverse dielectric tensor, which determines $\boldsymbol{D}(\boldsymbol{s})$ and $\boldsymbol{n}(\boldsymbol{s})$ as described in $\S 2$. It is simpler to represent the complex 3-vector $\boldsymbol{D}$ as a complex 2-vector $\boldsymbol{d}(\boldsymbol{s})$ perpendicular to $\boldsymbol{s}$, determined by the solution of Maxwell's equations (see $\S 3)$. $\boldsymbol{d}(\boldsymbol{s})$ fully describes the orientation and eccentricity of the polarization ellipse corresponding to the wave (1.1) - a connection apparently first understood by Gibbs (1928) (see also Hayes 1984). Several representations are convenient for $\boldsymbol{d}$ and $\boldsymbol{s}$ : polar coordinates, giving the $\theta$ and $\phi$ components of $\boldsymbol{d}$; stereographic coordinates $\boldsymbol{R}=\{X, Y\}$, giving the $X$ and $Y$ components of $\boldsymbol{d}$; a circular basis, specifying $\boldsymbol{d}$ in terms of its right- and left-handed components (in polar or stereographic coordinates). However, the representation we will emphasize, and make abundant use of, is the complex ratio of components of $\boldsymbol{d}$ (in any coordinates or basis but principally the circular basis), which contains all physical information.

In any basis, $\boldsymbol{d}$ and $\boldsymbol{n}$ are determined as the eigenvectors and eigenvalues of a $2 \times 2$ complex and usually non-Hermitian matrix $\boldsymbol{m}$. The complex combinations $Z=X+\mathrm{i} Y$ and $Z^{*}=X-\mathrm{i} Y$ enable $\boldsymbol{m}$ to be written in terms of several polynomials (quartic in $Z$ and $Z^{*}$ ) that are physically transparent (even when the crystal is not), leading to explicit forms for $\boldsymbol{d}$ and $\boldsymbol{n}$. The form we will use most-in a sense, the heart of the algebraic part of the paper - is equation (3.21) for the complex ratio of components.

The geometric heart of the paper is the description of three kinds of singularity (see $\S 4$ ). First are the degeneracies of $\boldsymbol{m}$ (see $\S 4 a$ ); for a non-chiral transparent crystal, these points in $s$-space are the optic axes; linear dichroism splits each optic axis into a pair of singular axes, which approach and coalesce again as chirality is increased. Singular axes and optic axes have very different geometries, reflecting the difference between eigenvectors of Hermitian and non-Hermitian matrices, studied 
recently in other contexts (Heiss 2000; Heiss \& Harney 2001; Rotter 2001, 2002; Berry 2003; Keck et al. 2003; Korsch \& Mossman 2003).

Second are the $C$ points in $s$-space: directions where $\boldsymbol{d}$ is purely circularly polarized (see $\S 4 b$ ). C points of the two polarizations are the zeros of polynomials in $Z$ and $Z^{*}$, and they are independent of chirality. In the absence of chirality, they coincide with the optic or singular axes, reflecting a known property of complex symmetric matrices, apparently due to Synge (1964) (see also Hayes 1984) and rediscovered by Heiss \& Harney (2001). When chirality is added, with constant absorption, the $\mathrm{C}$ points obey the haunting theorem: they remain fixed as the axes move and coalesce; in a sense they are ghosts that haunt the directions of departed singular axes. The $\mathrm{C}$ points are singularities of the pattern of axes of polarization ellipses in $s$-space, with index $+\frac{1}{2}$ (transparent non-chiral, transparent chiral or dichroic chiral) or $+\frac{1}{4}$ (dichroic non-chiral).

Third are the $L$ lines in $s$-space, where the polarization is purely linear (see $\S 4 c$ ). $\mathrm{L}$ lines separate the $\boldsymbol{R}$-plane into regions of right- and left-handed polarization.

When the wave direction is reversed $(s \rightarrow-s)$, the polarizations $\boldsymbol{d}$, and the associated singularities, possess antipodal symmetries that are interesting and not obvious; these are described in $\S 5$.

Much of the singular behaviour can be described simply in terms of a local model (see $\S 6$ ), in which the quartic polynomials are replaced by linear functions of $Z$ and $Z^{*}$. This describes a pair of singular axes and its coalescence into a single optic axis, and the associated $\mathrm{C}$ points and $\mathrm{L}$ lines.

The different types of singularity affect the interference fringes and brushes seen directly in $s$-space through a sheet of crystal with and without polarizing and analysing filters. In $\S 7$, simple general expressions are derived for the light intensities. We show simulations for different combinations of polarizer and analyser, and different combinations of anisotropy, absorption and chirality, chosen to illustrate the three types of singularity.

For a transparent non-chiral crystal, the matrix $\boldsymbol{m}$ is real symmetric. When chirality is added, $\boldsymbol{m}$ is complex Hermitian. With linear dichroism but not chirality, $\boldsymbol{m}$ is complex symmetric. For the most general case that we emphasize here, where the crystal possesses both absorption and chirality, $\boldsymbol{m}$ is neither real nor Hermitian. Then the two polarizations $\boldsymbol{d}$ are not orthogonal, and the right and left eigenvectors are different. Our analysis makes use of a number of elementary but not well-known matrix relations, which are collected in the appendix for convenience.

Although we use the convenient term 'crystal', the results apply to any anisotropic or chiral medium, for example, liquid crystals, or plastics such as overhead-projector transparency foil (Berry et al. 1999), provided its anisotropy is non-magnetic. (Magnetic anisotropy has been treated by Boulanger \& Hayes (1990), who also use complex vectors ('bivectors'), though of the form $\operatorname{Re} \boldsymbol{E}+\mathrm{i} \operatorname{Re} \boldsymbol{B}$ rather than $\boldsymbol{d}$.)

We need to make three points about notation and representation. First, we will not make use of the bra-ket notation reminiscent of quantum mechanics, namely $|\boldsymbol{d}\rangle$ for column vectors and $\langle\boldsymbol{d}|$ for the complex-conjugate row vectors, although this is sometimes convenient for transparent crystals (Berry \& Klein 1996). The reason is that the notation $\langle\boldsymbol{d}|$ might invite confusion between adjoint vectors and left eigenvectors, and this distinction is important in chiral dichroic crystals; moreover, we will seldom use row vectors. Therefore, we decided to use regular vector notation $\boldsymbol{d}$. Second, the representation of $\boldsymbol{d}$ as a 2-vector is reminiscent of the Jones calculus 
(see, for example, Azzam \& Bashara 1977; Brosseau 1998), but since we will employ several equivalent bases, any of which could with justification be called the Jones vector, we will not use this terminology. Third, although very effective use has been made of the Poincaré sphere (Series 1975; Ramachandran \& Ramaseshan 1961; Berry 1994) in understanding polarization states, we will not make explicit reference to this geometric construction. Rather, we will make abundant use of an equivalent representation: the stereographic projection of points from the Poincaré sphere to the complex plane of the ratio of components of $\boldsymbol{d}$ (see $\S 4 b$ and thereafter). (It is interesting to note that Poincaré arrived at his sphere by the reverse route: from the ratio of components, via inverse stereographic projection (Poincaré 1892, §158).)

\section{Dielectric tensor and the eigenpolarization equations}

The constitutive relation between the electric field $\boldsymbol{E}$ and displacement $\boldsymbol{D}$ vectors defines the reciprocal dielectric tensor $\boldsymbol{\eta}$,

$$
\boldsymbol{E}(s)=\frac{1}{\varepsilon_{0}} \boldsymbol{\eta}(\boldsymbol{s}) \cdot \boldsymbol{D}(\boldsymbol{s}) .
$$

In components, with

$$
\boldsymbol{D}=D_{x} \boldsymbol{e}_{x}+D_{y} \boldsymbol{e}_{y}+D_{z} \boldsymbol{e}_{z},
$$

$\boldsymbol{\eta}$ can be written

$$
\boldsymbol{\eta}(\boldsymbol{s})=\left(\begin{array}{ccc}
u_{x x} & u_{x y}-\mathrm{i} g_{z}(\boldsymbol{s}) & u_{x z}+\mathrm{i} g_{y}(\boldsymbol{s}) \\
u_{x y}+\mathrm{i} g_{z}(\boldsymbol{s}) & u_{y y} & u_{y z}-\mathrm{i} g_{x}(\boldsymbol{s}) \\
u_{x z}-\mathrm{i} g_{y}(\boldsymbol{s}) & u_{y z}+\mathrm{i} g_{x}(\boldsymbol{s}) & u_{z z}
\end{array}\right) .
$$

The symmetric part of $\boldsymbol{\eta}$ is the anisotropy tensor $\boldsymbol{u}=\left\{u_{x x}, \ldots\right\}$, describing the birefringence of the crystal. Here we will concentrate on the general case where the eigenvalues of $\boldsymbol{u}$ (principal dielectric constants) are all different, corresponding to a biaxial material; if two of the eigenvalues are the same, the material is uniaxialan elementary situation that will hardly feature in our analysis. For a transparent crystal, $\boldsymbol{u}$ is real; for a crystal with linear dichroism, $\boldsymbol{u}$ is complex. Absorption is guaranteed (that is, gain is avoided) if the imaginary part of each of the three eigenvalues is negative. For us, the interesting situation corresponds to a dichroic material where the eigenvalues of $\operatorname{Im} \boldsymbol{u}$ are different; the anisotropic components of $\operatorname{Im} \boldsymbol{u}$ can be positive or negative, with absorption being ensured by adding a sufficiently large constant diagonal part, $-\mathrm{i} C \delta_{i j}$.

In general, the principal axes of $\operatorname{Re} \boldsymbol{u}$ and $\operatorname{Im} \boldsymbol{u}$ are different. It will be convenient to choose coordinate axes along the principal axes of $\operatorname{Re} \boldsymbol{u}$, so that, denoting the (real) dielectric constants by $u_{1}, u_{2}, u_{3}$,

$$
\boldsymbol{u}=\left(\begin{array}{ccc}
u_{1} & 0 & 0 \\
0 & u_{2} & 0 \\
0 & 0 & u_{3}
\end{array}\right)+\mathrm{i}\left(\begin{array}{lll}
\operatorname{Im} u_{x x} & \operatorname{Im} u_{x y} & \operatorname{Im} u_{x z} \\
\operatorname{Im} u_{x y} & \operatorname{Im} u_{y y} & \operatorname{Im} u_{y z} \\
\operatorname{Im} u_{x z} & \operatorname{Im} u_{y z} & \operatorname{Im} u_{z z}
\end{array}\right), \quad u_{1} \geqslant u_{2} \geqslant u_{3} .
$$

(It is also possible to choose coordinate axes along the principal axes of the full complex $\boldsymbol{u}$, but these coordinates are generally complex, so we do not follow this route.)

Proc. R. Soc. Lond. A (2003) 
The antisymmetric part of $\boldsymbol{\eta}$ is determined by the optical activity vector $\boldsymbol{g}=$ $\left\{g_{x}, g_{y}, g_{z}\right\}$, describing the chirality (optical activity) of the crystal. Natural optical activity represents the simplest non-trivial non-local response of the crystal, corresponding to a contribution to $\boldsymbol{E}$ proportional to $\nabla \times \boldsymbol{D}$ (Landau et al. 1984). Thus $\boldsymbol{g}$ depends linearly on $\boldsymbol{s}$, the proportionality introducing a symmetric optical activity tensor $\gamma$,

$$
\boldsymbol{g}(\boldsymbol{s})=\boldsymbol{\gamma} \cdot \boldsymbol{s}=\left(\begin{array}{lll}
\gamma_{x x} & \gamma_{x y} & \gamma_{x z} \\
\gamma_{x y} & \gamma_{y y} & \gamma_{y z} \\
\gamma_{x z} & \gamma_{y z} & \gamma_{z z}
\end{array}\right)\left(\begin{array}{c}
s_{x} \\
s_{y} \\
s_{z}
\end{array}\right)
$$

For a material with circular dichroism, that is, absorption different for right- and left-handed polarizations, $\boldsymbol{\gamma}$ has an imaginary part. $\boldsymbol{u}$ and $\boldsymbol{\gamma}$ determine the optical properties of the crystal, and it can be constrained by the symmetry class of the crystal (Ramachandran \& Ramaseshan 1961; Nye 1995), though here we consider the general case.

Although we will concentrate on crystals with natural optical activity, we mention here that optical activity induced by an external magnetic field $\boldsymbol{H}$, that is, Faraday rotation, is also described by (2.3), but the analogue of the optical activity vector has the form $\boldsymbol{g}=\boldsymbol{\gamma} \cdot \boldsymbol{H}$ rather than (2.5) (Landau et al. 1984).

In the familiar case of a transparent isotropic optically active material, the general expressions reduce to

$$
u_{i j}=\delta_{i j} / n_{0}^{2}, \quad \gamma_{i j}=\Gamma \delta_{i j}, \quad \text { i.e. } g_{i}=\Gamma s_{i} .
$$

In later sections, we will give numerical illustrations of the optical singularities, for which it will be convenient to use a particular family of absorbing chiral crystals, with parameters sufficiently general to exhibit the various singularities and associated phenomena. This family, chosen after a number of numerical experiments (and not intended to represent any particular actual crystal), has isotropic chirality and anisotropy tensor, of the form (2.4), given by

$$
\begin{aligned}
\boldsymbol{u}= & \left(\begin{array}{ccc}
0.409338 & 0 & 0 \\
0 & 0.400577 & 0 \\
0 & 0 & 0.395554
\end{array}\right) \\
& +\mathrm{i} A\left(\begin{array}{ccc}
0.409338 & 0.025 & 0.03 \\
0.025 & 0.400577 & -0.022 \\
0.03 & -0.022 & 0.395554
\end{array}\right), \quad \gamma_{i j}=\Gamma \delta_{i j}, \quad \text { i.e. } g_{i}=\Gamma s_{i},
\end{aligned}
$$

with $A$ quantifying the strength of the absorption, and chirality quantified by $\Gamma$.

The 'crystal space' corresponding to (2.7) is the $A, \Gamma$-plane, whose two dimensions capture, as we will see, the essential behaviour of the singularities: behaviour stable under perturbations. The full crystal space has 15 dimensions: three for each of $\operatorname{Re} \boldsymbol{u}$, $\operatorname{Re} \gamma$ and $\operatorname{Im} \gamma$ and six for $\operatorname{Im} \boldsymbol{u}$. We will not emphasize the fact that the components of $\boldsymbol{u}$ and $\boldsymbol{\gamma}$ often vary significantly with the wavelength $\lambda$ (pleochroism), but note that varying $\lambda$ can provide a physically convenient one-dimensional path through crystal space.

Although our formulae will be written for the general case of anisotropic chirality tensor $\gamma$, we will argue later that this introduces no essentially different phenomena from those occurring in crystals with isotropic chirality and anisotropic complex $\boldsymbol{u}$, as in (2.7).

Proc. R. Soc. Lond. A (2003) 


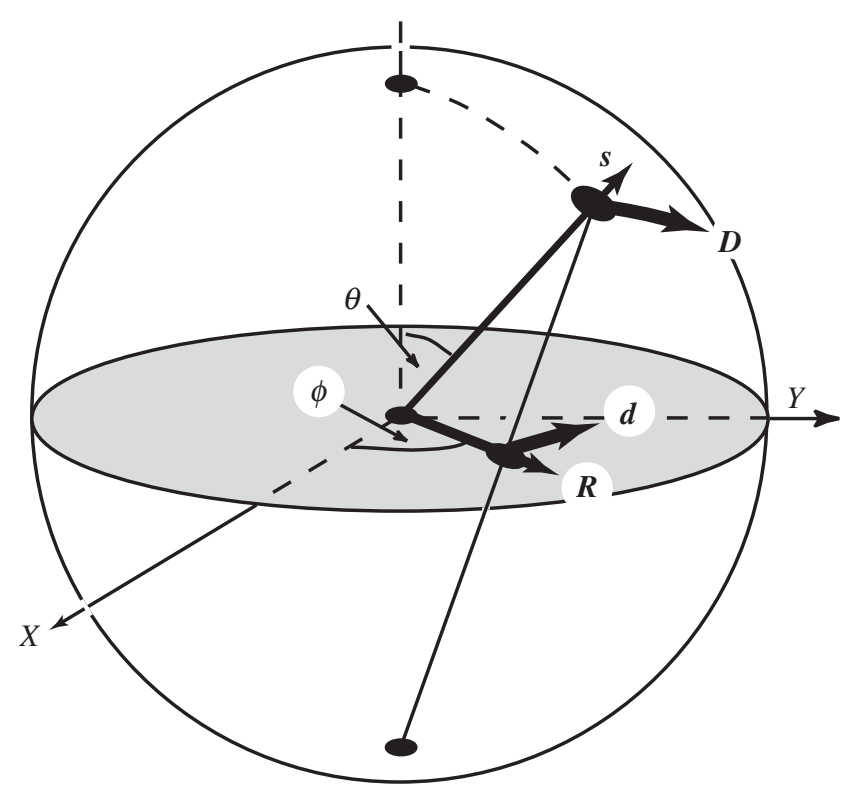

Figure 1. South-pole stereographic projection from direction $s$ on the unit sphere with polar coordinates $\theta, \phi$, to vector $\boldsymbol{R}$ in the plane, with coordinates $X, Y$; electric displacement vector $\boldsymbol{D}$, perpendicular to $\boldsymbol{s}$ and usually complex, but here depicted as real, projects to vector $\boldsymbol{d}$. The shaded disk is the projection of the northern hemisphere.

With the wave (1.1) and the constitutive relation (2.1), some elementary manipulations enable Maxwell's equations to be transformed into

$$
-\boldsymbol{s} \times(\boldsymbol{s} \times(\boldsymbol{\eta} \cdot \boldsymbol{D}(\boldsymbol{s})))=\boldsymbol{\eta} \cdot \boldsymbol{D}(\boldsymbol{s})-\boldsymbol{s} \cdot \boldsymbol{\eta} \cdot \boldsymbol{D}(\boldsymbol{s}) \boldsymbol{s}=\frac{1}{n^{2}(\boldsymbol{s})} \boldsymbol{D}(\boldsymbol{s}) .
$$

Obviously, $\boldsymbol{D} \cdot \boldsymbol{s}=0$, that is, $\boldsymbol{D}$ is transverse. This can be written as a matrix equation,

$$
\boldsymbol{M}(s) \cdot \boldsymbol{D}(s)=\lambda(s) \boldsymbol{D}(s) .
$$

The following known properties of the matrix $\boldsymbol{M}$ (see, for example, Ramachandran \& Ramaseshan 1961) are easily confirmed using (2.3)-(2.5), after incorporating $\boldsymbol{D} \cdot \boldsymbol{s}=0$ : for a transparent non-chiral crystal, $\boldsymbol{M}$ is real symmetric; for a transparent chiral crystal, $\boldsymbol{M}$ is complex Hermitian; for a dichroic non-chiral crystal, $\boldsymbol{M}$ is complex symmetric; and for a dichroic chiral crystal, $\boldsymbol{M}$ is complex non-Hermitian.

Transversality implies that $\operatorname{det} \boldsymbol{M}=0$, so that one of the eigenvalues $\lambda$ is zero. The other two eigenvalues determine the refractive indices by $\lambda=1 / n^{2}$, and the corresponding eigenvectors are the polarizations. Thus crystal optics depends on the $2 \times 2$ part of $\boldsymbol{M}$ transverse to $\boldsymbol{s}$, as we now elaborate.

\section{Explicit formulae for eigenpolarizations}

With the direction $s$ specified by polar coordinates $\theta, \phi$, the 3 -vector $\boldsymbol{D}$, being transverse, can be written as the 2-vector $\boldsymbol{d}$,

$$
\boldsymbol{D}=d_{\theta} \boldsymbol{e}_{\theta}+d_{\phi} \boldsymbol{e}_{\phi}, \quad \boldsymbol{d}_{\mathrm{polar}}=\left(\begin{array}{c}
d_{\theta} \\
d_{\phi}
\end{array}\right) .
$$


More convenient will be coordinates defined by south-pole stereographic projection from the $s$ sphere to the equatorial plane (figure 1); these coordinates are

$$
\left.\begin{array}{rl}
\boldsymbol{R} & =\{X, Y\}=\frac{\left(1-s_{z}\right)}{1-s_{z}^{2}}\left\{s_{x}, s_{y}\right\}=\tan \frac{1}{2} \theta\{\cos \phi, \sin \phi\}, \\
R & =\sqrt{X^{2}+Y^{2}}=\tan \frac{1}{2} \theta=\sqrt{\frac{1-s_{z}}{1+s_{z}}}, \\
s & =\left\{s_{x}, s_{y}, s_{z}\right\}=\frac{1}{1+R^{2}}\left\{2 X, 2 Y, 1-R^{2}\right\} .
\end{array}\right\}
$$

It will often be helpful to represent the stereographic vector $\boldsymbol{R}$ in terms of the complex variables

$$
Z=X+\mathrm{i} Y \quad \text { and } \quad Z^{*}=X-\mathrm{i} Y .
$$

Later we will require the stereographic representation of the antipodal transformation $s \rightarrow-s$, namely,

$$
s \rightarrow-s \quad \Rightarrow \quad R \rightarrow-\frac{R}{R^{2}}, \quad \text { i.e. } Z \rightarrow-\frac{1}{Z^{*}} .
$$

The corresponding components of $\boldsymbol{d}$ (figure 1) are

$$
\boldsymbol{d}_{\text {stereographic }}=\left(\begin{array}{l}
d_{X} \\
d_{Y}
\end{array}\right)=\left(\begin{array}{cc}
\cos \phi & -\sin \phi \\
\sin \phi & \cos \phi
\end{array}\right)\left(\begin{array}{l}
d_{\theta} \\
d_{\phi}
\end{array}\right)
$$

It will also be useful to represent $\boldsymbol{d}$ in a circular basis, defined by its right- and left-handed polarization components,

$$
\boldsymbol{d}_{\text {circular }}=\left(\begin{array}{c}
d_{\mathrm{R}} \\
d_{\mathrm{L}}
\end{array}\right)=\frac{1}{\sqrt{2}}\left(\begin{array}{cc}
1 & -\mathrm{i} \\
1 & \mathrm{i}
\end{array}\right)\left(\begin{array}{l}
d_{X} \\
d_{Y}
\end{array}\right)
$$

The two polarizations $\boldsymbol{d}^{ \pm}$, and the corresponding eigenvalues $\lambda^{ \pm}$, are determined by the $2 \times 2$ transverse part $\boldsymbol{m}$ of the matrix $\boldsymbol{M}$,

$$
\boldsymbol{m}(\boldsymbol{R}) \cdot \boldsymbol{d}^{ \pm}(\boldsymbol{R})=\lambda^{ \pm}(\boldsymbol{R}) \boldsymbol{d}^{ \pm}(\boldsymbol{R}) .
$$

In polar coordinates,

$$
\boldsymbol{m}_{\mathrm{polar}}(\boldsymbol{R})=\left(\begin{array}{ll}
\boldsymbol{M}_{\theta \theta}(s) & \boldsymbol{M}_{\theta \phi}(s) \\
\boldsymbol{M}_{\phi \theta}(\boldsymbol{s}) & \boldsymbol{M}_{\phi \phi}(\boldsymbol{s})
\end{array}\right)
$$

In stereographic coordinates,

$$
\boldsymbol{m}_{\text {stereographic }}(\boldsymbol{R})=\left(\begin{array}{cc}
\cos \phi & -\sin \phi \\
\sin \phi & \cos \phi
\end{array}\right) \boldsymbol{m}_{\text {polar }}\left(\begin{array}{cc}
\cos \phi & \sin \phi \\
-\sin \phi & \cos \phi
\end{array}\right)
$$

and in the circular basis,

$$
\boldsymbol{m}_{\text {circular }}(\boldsymbol{R})=\frac{1}{2}\left(\begin{array}{cc}
\exp (-\mathrm{i} \phi) & -\mathrm{i} \exp (-\mathrm{i} \phi) \\
\exp (\mathrm{i} \phi) & \mathrm{i} \exp (\mathrm{i} \phi)
\end{array}\right) \boldsymbol{m}_{\text {polar }}(\boldsymbol{R})\left(\begin{array}{cc}
\exp (\mathrm{i} \phi) & \exp (-\mathrm{i} \phi) \\
\mathrm{i} \exp (\mathrm{i} \phi) & -\mathrm{i} \exp (-\mathrm{i} \phi)
\end{array}\right) .
$$

Where normalization is relevant, we will always employ the complex scalar product,

$$
\boldsymbol{d}^{ \pm *} \cdot \boldsymbol{d}^{ \pm}=1
$$

Proc. R. Soc. Lond. A (2003) 
The transverse matrix $\boldsymbol{m}$ takes its simplest form in the circular basis. An elementary though lengthy calculation, rotating $\boldsymbol{M}$ in (2.8) and (2.9) to polar coordinates according to (3.8), and then further transforming according to (3.9) and (3.10), leads to

$$
\boldsymbol{m}_{\text {circular }}=\frac{1}{2\left(1+R^{2}\right)^{2}}\left[Q\left(\begin{array}{ll}
1 & 0 \\
0 & 1
\end{array}\right)+\left(\begin{array}{cc}
0 & P_{2} \\
P_{1} & 0
\end{array}\right)+G\left(\begin{array}{cc}
1 & 0 \\
0 & -1
\end{array}\right)\right],
$$

involving a multiple of the unit matrix and diagonal and off-diagonal traceless matrices, and the following four polynomials.

Most important are the two principal polynomials $P_{1}$ and $P_{2}$, in the off-diagonal part of the traceless contribution to $\boldsymbol{m}_{\text {circular }}$, depending on the anisotropy tensor $\boldsymbol{u}$,

$$
\begin{aligned}
P_{1}(Z, \boldsymbol{u})= & \left(1+Z^{4}\right)\left(u_{x x}-u_{y y}\right)+2 Z^{2}\left(2 u_{z z}-u_{x x}-u_{y y}\right) \\
& \quad+2 \mathrm{i}\left(1-Z^{4}\right) u_{x y}-4 Z\left(1-Z^{2}\right) u_{x z}-4 \mathrm{i} Z\left(1+Z^{2}\right) u_{y z} \\
= & P_{1}(Z, \operatorname{Re} \boldsymbol{u})+\mathrm{i} P_{1}(Z, \operatorname{Im} \boldsymbol{u})
\end{aligned}
$$

and

$$
\begin{aligned}
P_{2}(Z, \boldsymbol{u})= & \left(1+Z^{* 4}\right)\left(u_{x x}-u_{y y}\right)+2 Z^{* 2}\left(2 u_{z z}-u_{x x}-u_{y y}\right) \\
& -2 \mathrm{i}\left(1-Z^{* 4}\right) u_{x y}-4 Z^{*}\left(1-Z^{* 2}\right) u_{x z}+4 \mathrm{i} Z^{*}\left(1+Z^{* 2}\right) u_{y z} \\
= & {\left[P_{1}\left(Z, \boldsymbol{u}^{*}\right)\right]^{*}=\left[P_{1}(Z, \operatorname{Re} \boldsymbol{u})\right]^{*}+\mathrm{i}\left[P_{1}(Z, \operatorname{Im} \boldsymbol{u})\right]^{*} }
\end{aligned}
$$

(note that the sign of $\mathrm{i}$ in the second equality in (3.14) is not changed). If the crystal is transparent $(\boldsymbol{u}$ real $)$, then $P_{2}=P_{1}^{*}$ and this part of $\boldsymbol{m}$ is Hermitian. For an isotropic crystal (absorbing or not), $P_{1}=P_{2}=0$.

The polynomial $G$ multiplying the diagonal part of the traceless contribution to $\boldsymbol{m}_{\text {circular }}$ depends on the optical activity tensor of the crystal, defined in (2.5),

$$
\begin{aligned}
G(\boldsymbol{R}, \boldsymbol{\gamma})= & 2\left(1+R^{2}\right)^{2} \boldsymbol{g}(\boldsymbol{s}) \cdot \boldsymbol{s} \\
=2\left[4 X^{2} \gamma_{x x}+\right. & 4 Y^{2} \gamma_{y y}+\left(1-R^{2}\right)^{2} \gamma_{z z} \\
& \left.\quad+8 X Y \gamma_{x y}+4\left(1-R^{2}\right)\left(X \gamma_{x z}+Y \gamma_{x z}\right)\right] .
\end{aligned}
$$

If the optical activity is isotropic, then, $G=2 \Gamma\left(1+R^{2}\right)^{2}$ (cf. (2.6)), where $\Gamma$ is real if the crystal is transparent and complex if it possesses circular dichroism.

The polynomial $Q$ multiplying the unit matrix, which determines the average $\frac{1}{2}\left(\lambda^{+}+\lambda^{-}\right)$of the two eigenvalues, depends on the anisotropy tensor $\boldsymbol{u}$,

$$
\begin{aligned}
Q(\boldsymbol{R}, \boldsymbol{u})=(1+ & \left.R^{4}\right)\left(u_{x x}+u_{y y}\right)+2\left(Y^{2}-X^{2}\right)\left(u_{x x}-u_{y y}\right) \\
& +4 R^{2} u_{z z}-8 X Y u_{x y}-4 X\left(1-R^{2}\right) u_{x z}-4 Y\left(1-R^{2}\right) u_{y z} .
\end{aligned}
$$

From (3.12), the eigenvalues of $\boldsymbol{m}$ are

$$
\lambda^{ \pm}(\boldsymbol{R})=\frac{1}{2\left(1+R^{2}\right)^{2}}\left[Q \pm \sqrt{P_{1} P_{2}+G^{2}}\right] .
$$

Since the argument of the square-root function is complex when there is absorption, it is necessary to specify the branch. Here and hereafter, the square root of any quantity $F$ will be defined by

$$
\sqrt{F}=|\sqrt{F}| \exp \left(\frac{1}{2} \mathrm{i} \arg F\right), \quad|\arg F| \leqslant \pi,
$$

Proc. R. Soc. Lond. A (2003) 

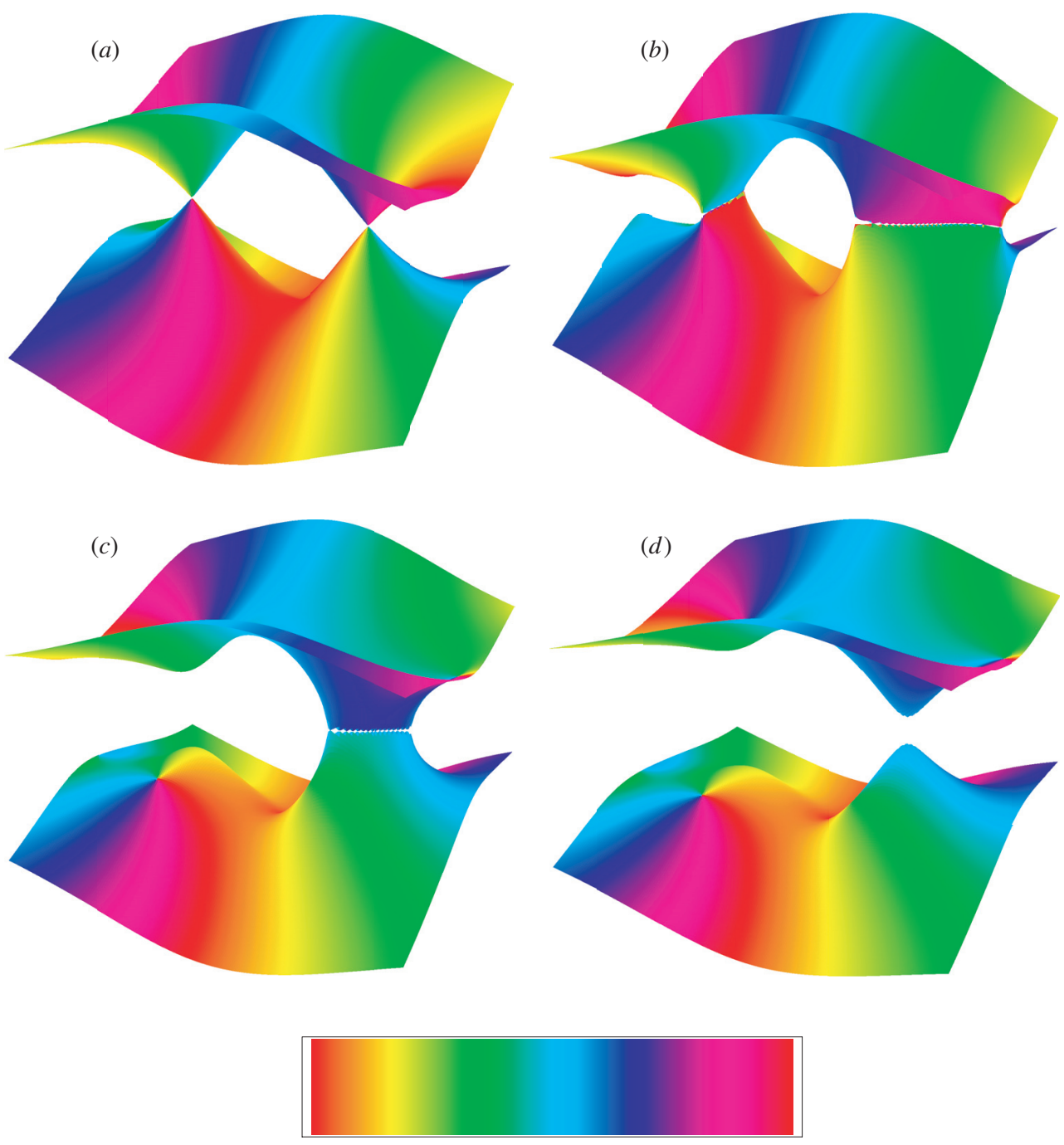

0

phase

$2 \pi$

Figure 2. Real parts of eigenvalues $\lambda^{ \pm}$, computed from (3.17) with $Q=0$, represented as sheets above the $\boldsymbol{R}$-plane for $|X|<1,|Y|<1$ (i.e. including the northern hemisphere $R<1$ ), for the dielectric tensor (2.7), for (a) $A=\Gamma=0$ (transparent non-chiral); (b) $A=0.1, \Gamma=0$ (absorbing non-chiral); (c) $A=0.1, \Gamma=0.0025$ (absorbing chiral); $(d) A=0.1, \Gamma=0.0035$ (chirality dominated). The sheets are connected at the degeneracies (see $\S 4 a$ ): optic axes in $(a)$, singular axes in $(b)$ and $(c)$. The sheets are colour coded as indicated, with hue representing $\arg w_{\text {circular }}$ (equation $(3.21)$ ), showing $\mathrm{C}$ points where all colours meet (see $\left.\S 4 b\right)$.

so that $\operatorname{Re} \sqrt{F}=0$ and the $F$-plane is cut along the negative real axis, where $\operatorname{Im} \sqrt{F}$ has a discontinuity of $2 \mid \sqrt{F}$. The two refractive indices are

$$
n^{ \pm}(\boldsymbol{R})=\frac{1}{\sqrt{\lambda^{ \pm}(\boldsymbol{R})}}=\bar{n} \pm \frac{1}{2} \Delta n \approx \sqrt{\frac{2}{Q}}\left(1+R^{2}\right)\left[1 \mp \frac{\sqrt{P_{1} P_{2}+G^{2}}}{2 Q}\right]
$$

Proc. R. Soc. Lond. A (2003) 
where the approximation holds for the common situation where the traceless contributions $P_{1}, P_{2}$ and $G$ are much smaller than $Q$. Figure 2 shows the two eigenvalue sheets for several crystals.

The polarization states are

$$
\boldsymbol{d}_{\text {circular }}^{ \pm}=N_{ \pm}\left(\begin{array}{c}
P_{2} \\
\pm \sqrt{P_{1} P_{2}+G^{2}}-G
\end{array}\right)=N_{ \pm}\left(\begin{array}{c} 
\pm \sqrt{P_{1} P_{2}+G^{2}}+G \\
P_{1}
\end{array}\right)
$$

where here and hereafter $N_{ \pm}$will denote unspecified normalization constants determined by (3.11).

In the next section we will see that the essential physics of $\boldsymbol{d}^{ \pm}$, for which normalization and overall phase are irrelevant, is captured by the ratio of components of these eigenvectors, that is, by

$$
w_{\text {circular }}^{ \pm}=\frac{d_{\mathrm{L}}^{ \pm}}{d_{\mathrm{R}}^{ \pm}}=\frac{P_{1}}{ \pm \sqrt{P_{1} P_{2}+G^{2}}+G}=\frac{ \pm \sqrt{P_{1} P_{2}+G^{2}}-G}{P_{2}} .
$$

The complex number $w$ is the point representing the polarization of the light in the plane obtained by south-pole stereographic projection of the Poincaré sphere (Azzam \& Bashara 1977; Brosseau 1998). As we will see, the phase $\arg w$ represents the orientation of the polarization ellipse, and the modulus $|w|$ determines the eccentricity. All information about the optics of a crystal is contained in the eigenvalues $\lambda^{ \pm}$and the mapping from the plane of directions $\boldsymbol{R}$ (or $Z$ or $Z^{*}$ ) to the complex functions $w_{\text {circular }}^{ \pm}(Z)$; the two sheets + and - may be separated (if $G$ is large enough, as explained later) or connected at branch points where the square root vanishes (figure 2).

In stereographic coordinates, the matrix $\boldsymbol{m}$, given by (3.9), is

$$
\boldsymbol{m}_{\text {stereographic }}=\frac{1}{4\left(1+R^{2}\right)^{2}}\left(\begin{array}{cc}
2 Q+P_{1}+P_{2} & -\mathrm{i}\left(P_{1}-P_{2}\right)-2 \mathrm{i} G \\
-\mathrm{i}\left(P_{1}-P_{2}\right)+2 \mathrm{i} G & 2 Q-\left(P_{1}+P_{2}\right)
\end{array}\right) .
$$

It is easily verified that $\boldsymbol{m}_{\text {stereographic }}$ inherits the symmetries of $\boldsymbol{M}$ listed after (2.9). The corresponding polarization states are

$$
\boldsymbol{d}_{\text {stereographic }}^{ \pm}=N_{ \pm}\left(\begin{array}{c}
-2 \mathrm{i} G-\mathrm{i}\left(P_{1}-P_{2}\right) \\
\pm 2 \sqrt{P_{1} P_{2}+G^{2}}-\left(P_{1}+P_{2}\right)
\end{array}\right)
$$

and the ratio of components is

$$
w_{\text {stereographic }}^{ \pm}=\frac{d_{Y}^{ \pm}}{d_{X}^{ \pm}}=\frac{\left(P_{1}+P_{2}\right) \mp 2 \sqrt{P_{1} P_{2}+G^{2}}}{\mathrm{i}\left(P_{1}-P_{2}+2 G\right)} .
$$

When the crystal is dichroic, so that the matrices (3.12) and (3.22) are nonHermitian, the two polarizations are not orthogonal: $\boldsymbol{d}^{-*} \cdot \boldsymbol{d}^{+} \neq 0$. (The states are always biorthogonal, as described in the appendix and applied in $\S 7$.)

\section{Singularities}

(a) Degeneracies: optic axes and singular axes

According to (3.17), the two refractive indices coincide when

$$
P_{1} P_{2}+G^{2}=0
$$

Proc. R. Soc. Lond. A (2003) 
The nature of these degeneracies depends on whether the crystal is transparent ( $\boldsymbol{m}$ Hermitian) or absorbing ( $\boldsymbol{m}$ non-Hermitian).

For a transparent crystal, $P_{1} P_{2}=\left|P_{1}\right|^{2}$ and $G$ is real, so degeneracies cannot occur unless the crystal is non-chiral, that is, $G=0$. Then the degeneracies are isolated points (codimension 2) in $\boldsymbol{R}$-space, at the zeros of the complex quartic polynomials $P_{1}$ or $P_{2}$. In this case, where $\boldsymbol{u}$ is real, it follows from (3.13) and (3.14) that the zeros of $P_{1}$ and $P_{2}$ are the same, so there are four zeros. These are the optic axes (Ramachandran \& Ramaseshan 1961; Born \& Wolf 1959): two in the northern hemisphere (figure $2 a$ ) and two in the southern hemisphere. (This slight departure from conventional terminology - referring to these four directions as four axes rather than two-will be convenient later.) It will become clear that the optic axes retain a significance when the crystal is chiral and/or dichroic, so it is convenient to define these in the general case, by

$$
\text { optic axes: } \quad P_{1}(Z, \operatorname{Re} \boldsymbol{u})=0 .
$$

Each optic axis is a double zero of $P_{1} P_{2}$, corresponding to the diabolical (doublecone) connection between the eigenvalue sheets, characteristic of Hermitian matrices (Berry \& Wilkinson 1984). In terms of the principal dielectric constants defined by (2.4), the optic axes lie on the $X$-axis in $\boldsymbol{R}$-space, at

$$
Z= \pm X_{0}, \pm \frac{1}{X_{0}}
$$

where

$$
X_{0}=\frac{\sqrt{u_{1}-u_{3}}-\sqrt{u_{2}-u_{3}}}{\sqrt{u_{1}-u_{2}}} .
$$

In the special case of a uniaxial crystal, where $u_{1}=u_{2}$ or $u_{2}=u_{3}$, the four optic axes degenerate into two.

If the crystal is absorbing but non-chiral, the degeneracies are still the zeros of the principal polynomials, but now the zeros of $P_{1}$ and $P_{2}$ do not coincide, so $P_{1} P_{2}$ possesses eight zeros, still isolated (that is, still with codimension 2). These are the singular axes (Ramachandran \& Ramaseshan 1961): branch points of the square roots in (3.17), characteristic of non-Hermitian matrices. Figure $2 b$ shows four singular axes in the northern hemisphere. Although the zeros of $P_{1}$ and $P_{2}$ do not coincide, it is easily confirmed that they are related by the antipodal transformation (3.4),

$$
\left.\begin{array}{l}
P_{1}=0: \quad Z=Z_{11}, Z_{12}, Z_{13}, Z_{14}, \\
P_{2}=0: \quad Z=-\frac{1}{Z_{11}^{*}},-\frac{1}{Z_{12}^{*}},-\frac{1}{Z_{13}^{*}},-\frac{1}{Z_{14}^{*}} .
\end{array}\right\}
$$

As the absorbing part $\operatorname{Im} \boldsymbol{u}$ of the anisotropy tensor is reduced to zero, the pairs of singular axes approach and coalesce onto the optic axes.

Singular axes are connected in pairs by branch cuts, whose locations are determined by (3.18). These connections are clearly visible in parts $(b)$ and $(c)$ of figure 2 . Across each cut, $\arg w$ jumps by $\pi$; the jumps are clearly visible in the hue-coded representations of $\arg w$ in figure 3 , as are the smooth phase connections between the + and - sheets.

Since $\boldsymbol{m}$ is non-Hermitian, the two polarizations $\boldsymbol{d}^{ \pm}$are non-orthogonal, that is, $\boldsymbol{d}^{ \pm *} \cdot \boldsymbol{d}^{\mp} \neq 0$. This aspect of non-hermiticity is extreme at the singular axes, where

Proc. R. Soc. Lond. A (2003) 

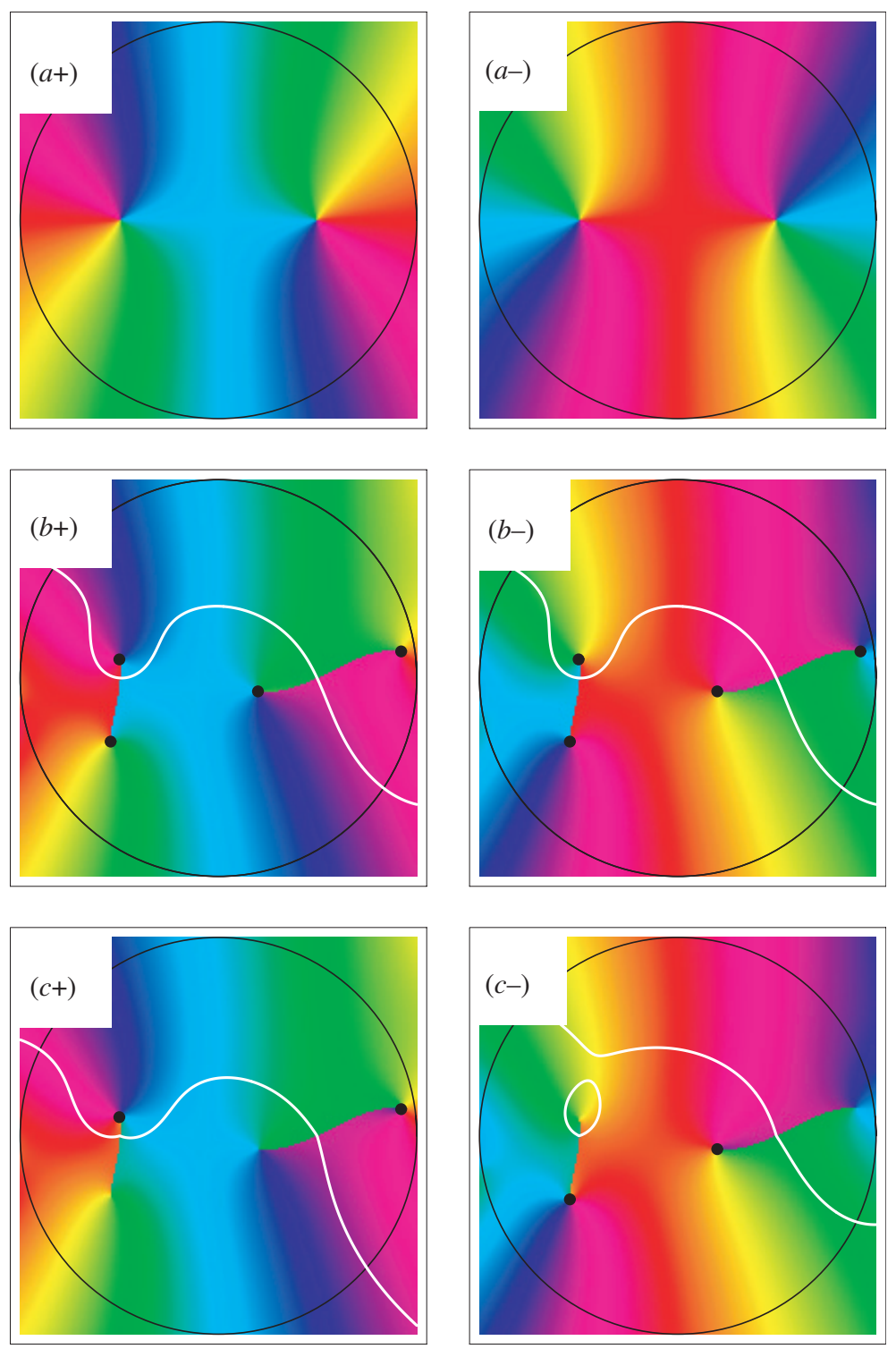

Figure 3. $(a+)-(f+) \arg w^{+},(a-)-(f-) \arg w^{-}$, colour coded as in figure 2, in the northern hemisphere $R<1$ (within the circle shown), for the crystal (2.7), for (a) $A=\Gamma=0$; (b) $A=0.1$, $\Gamma=0 ;(c) A=0.1, \Gamma=0.0005$. Black dots denote $\mathrm{C}$ points, and white lines denote $\mathrm{L}$ lines (see $\S 4 c)$. In $(b)-(e)$, the colours jump across the branch cuts.

the two polarizations coincide, that is, $\boldsymbol{d}^{+} \rightarrow \boldsymbol{d}^{-}$(as is obvious from equations (3.20), (3.21), (3.23) or (3.24)). It gives rise to interesting physics, as we will see.

In the fully general case, where chirality is present $(G \neq 0)$ as well as absorption, all eight branch-point degeneracies, that is, the singular axes, persist if the magnitude of $G$ is not too large; we call this the absorption-dominated regime. If there is too much chirality, the singular axes coincide and disappear, and the two eigenvalue sheets are fully separated (figure $2 d$ ); we call this the chirality-dominated regime. To see that 

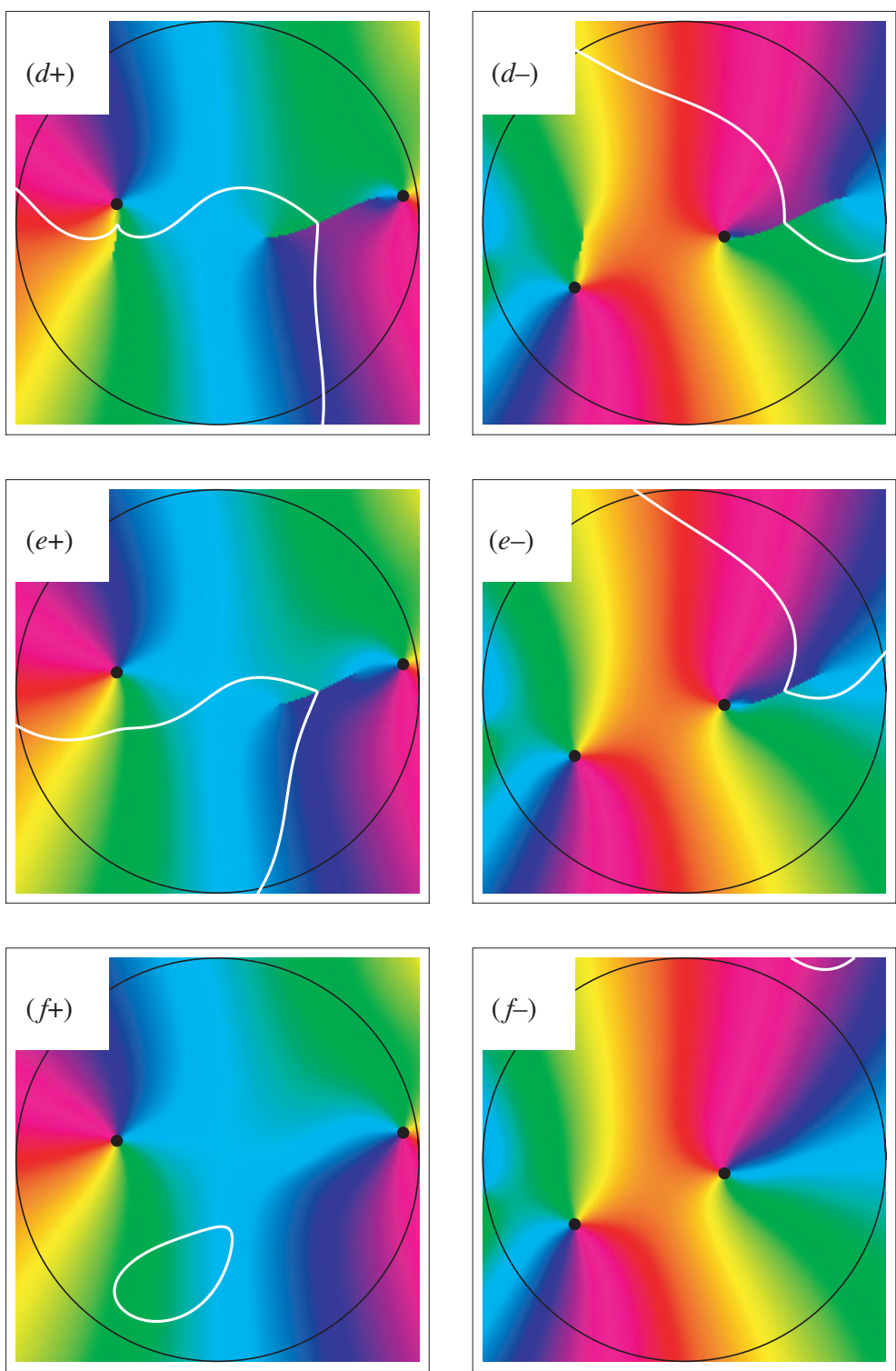

Figure 3. (Cont.) $(a+)-(f+) \arg w^{+},(a-)-(f-) \arg w^{-}$, colour coded as in figure 2 , in the northern hemisphere $R<1$ (within the circle shown), for the crystal (2.7), for $(d) A=0.1$, $\Gamma=0.0015 ;(e) A=0.1, \Gamma=0.0025 ;(f) A=0.1, \Gamma=0.0038$. Black dots denote C points, and white lines denote $\mathrm{L}$ lines (see $\S 4 c)$. In $(b)-(e)$, the colours jump across the branch cuts.

chirality domination must occur, we note that with the explicit forms (3.13), (3.14), the degeneracy condition (4.1) becomes

$$
\left.\begin{array}{r}
\left|P_{1}(Z, \operatorname{Re} \boldsymbol{u})\right|^{2}-\left|P_{1}(Z, \operatorname{Im} \boldsymbol{u})\right|^{2}+\operatorname{Re} G(\boldsymbol{R})^{2}-\operatorname{Im} G(\boldsymbol{R})^{2}=0, \\
\operatorname{Re}\left[P_{1}(Z, \operatorname{Re} \boldsymbol{u}) P_{1}(Z, \operatorname{Im} \boldsymbol{u})^{*}\right]+\operatorname{Re} G(\boldsymbol{R}) \operatorname{Im} G(\boldsymbol{R})=0 .
\end{array}\right\}
$$

If $\operatorname{Re} G$ is sufficiently large, the first of these equations has no solution, so there are no singular axes. In general, the two regimes are separated by a transition regime, 
where four singular axes (two antipodal pairs) have disappeared but four remain (figure $2 c$ shows an example). As pointed out by Ramachandran \& Ramaseshan (1961), amethyst is strongly pleochroic, and can be made absorption dominated or chirality dominated by changing the wavelength.

In the case of isotropic chirality without circular dichroism, that is, $G=2 \Gamma(1+$ $\left.R^{2}\right)^{2}$ with $\Gamma$ real, the four optic axes (4.3) and (4.4), satisfying (4.2), can satisfy the degeneracy condition for finite $\Gamma$ and $\operatorname{Im} \boldsymbol{u}$ as well as in the familiar non-chiral transparent case $(\Gamma=0, \operatorname{Im} \boldsymbol{u}=\mathbf{0})$. For this to happen, the chirality must take one of the critical values

$$
\Gamma_{\mathrm{c} 1}(\boldsymbol{u})= \pm \frac{\left|P_{1}\left(X_{0}, \operatorname{Im} \boldsymbol{u}\right)\right|}{2\left(1+X_{0}^{2}\right)^{2}}, \quad \Gamma_{\mathrm{c} 2}(\boldsymbol{u})= \pm \frac{\left|P_{1}\left(-X_{0}, \operatorname{Im} \boldsymbol{u}\right)\right|}{2\left(1+X_{0}^{2}\right)^{2}} .
$$

$\Gamma_{\mathrm{c} 1}$ corresponds to the optic axes $X_{0},-1 / X_{0}$, and $\Gamma_{\mathrm{c} 2}$ corresponds to the optic axes $-X_{0}, 1 / X_{0}$. The significance of this return by a singular axis to the direction of an original optic axis will be explained in $\S 4 c$.

If, in addition, the symmetry is such that principal axes of all three tensors $(\operatorname{Re} \boldsymbol{u}$, $\operatorname{Im} \boldsymbol{u}$ and $\gamma$ ) coincide, then, as a short argument shows, this return also corresponds to the simultaneous disappearance of all pairs of singular axes, that is, to the transition (in this case sudden) from absorption- to chirality-dominated regimes. After some reduction, the critical values (4.7) for this special but important case can be written in the more explicit form

$$
\Gamma_{\mathrm{c} 1}(\boldsymbol{u})=\Gamma_{\mathrm{c} 2}(\boldsymbol{u})=\frac{ \pm\left[u_{1} \operatorname{Im}\left(u_{y y}-u_{z z}\right)+u_{2} \operatorname{Im}\left(u_{z z}-u_{x x}\right)+u_{3} \operatorname{Im}\left(u_{x x}-u_{y y}\right)\right]}{2\left(u_{1}-u_{3}\right)} .
$$

\section{(b) $C$ (circular polarization) points and the haunting theorem}

In general, the polarizations $\boldsymbol{d}^{ \pm}$are elliptical. For certain directions $\boldsymbol{R}$, however, the polarizations are purely right-handed $\left(d_{\mathrm{L}}=0\right)$ or purely left-handed $\left(d_{\mathrm{R}}=0\right)$. These are the C points (Nye 1983a; Berry \& Dennis 2001; Dennis 2002; Berry 2001), alternatively characterized by the vector $\boldsymbol{d}_{\text {stereographic }}$ or $\boldsymbol{d}_{\text {polar }}$ being nilpotent. Thus the conditions for a $\mathrm{C}$ point can be written as

$$
\boldsymbol{d}_{\text {stereographic }} \cdot \boldsymbol{d}_{\text {stereographic }}=2 d_{\mathrm{R}} d_{\mathrm{L}}=0 \quad \Rightarrow \quad w_{\text {circular }}=0 \text { or } \infty .
$$

From (3.21), the $\mathrm{C}$ points are the zeros of the principal polynomials,

$$
\left.\begin{array}{lll}
P_{1}(Z, \boldsymbol{u})=0 & \Rightarrow & \text { C point of } \boldsymbol{d}^{+}, \mathrm{R} \text { type }\left(d_{\mathrm{L}}=0\right), \\
P_{2}(Z, \boldsymbol{u})=0 \quad \Rightarrow \quad & \text { C point of } \boldsymbol{d}^{-}, \mathrm{L} \text { type }\left(d_{\mathrm{R}}=0\right) .
\end{array}\right\}
$$

Thus there are four C points of $\boldsymbol{d}^{+}$and four of $\boldsymbol{d}^{-}$. At a $\mathrm{C}$ point (a zero or pole of $w$ ), the phase $\arg w$ is singular. Therefore, C points show up clearly when $\arg w$ is colour coded by hue, as in figures 2 and 3 .

Note that these conditions are independent of the chirality tensor $\gamma$. If $\boldsymbol{\gamma}=\mathbf{0}$, the $\mathrm{C}$ points coincide with the singular axes, at each of which the two polarizations have the same handedness: both $\boldsymbol{d}^{+}$and $\boldsymbol{d}^{-}$are of $\mathrm{R}$ type if $P_{1}=0$ and of $\mathrm{L}$ type if $P_{2}=0$ (the relation (4.10) is degenerate).

If now chirality is increased from zero, with fixed anisotropy tensor $\boldsymbol{u}$, so that the singular axes approach in pairs, the $\mathrm{C}$ points (one per pair) remain fixed, according 
to (4.10). We call this result the haunting theorem, since, in a sense, the $\mathrm{C}$ points are ghosts, haunting the positions of departed singular axes. In this general case, the singular axes are not $\mathrm{C}$ points, and the elliptic polarizations at each axis, being identical, share the same handedness. Even in the chirality-dominated regime, when there are no singular axes, the $\mathrm{C}$ points continue to haunt the directions $Z$ where the axes were for the non-chiral crystal. $\mathrm{C}$ points where there are no singular axes can be seen in parts $(c)$ and $(d)$ of figure 2 and parts $(d)-(f)$ of figure 3 . In the special case of a transparent crystal, whose degeneracies disappear in the presence of chirality, the $\mathrm{C}$ points haunt the departed optic axes (4.3) and (4.4).

In addition to handedness (right or left), describing the state of polarization at the $\mathrm{C}$ point itself, each $\mathrm{C}$ point is singular in a different sense: it possesses a topological index, describing the polarization ellipses in its neighbourhood. The index is the number of rotations of the axes of the ellipses in a circuit of the $\mathrm{C}$ point. To calculate the index, we note that, in the circular basis, the major axis direction $\phi_{\text {ellipse }}$ is given by the phases of the components, according to

$$
\boldsymbol{d}_{\text {circular }}=\left(\begin{array}{c}
\cos \frac{1}{2} \mu \exp \left(-\mathrm{i} \phi_{\text {ellipse }}\right) \\
\sin \frac{1}{2} \mu \exp \left(\mathrm{i} \phi_{\text {ellipse }}\right)
\end{array}\right) .
$$

Here, $\mu$, which describes the eccentricity of the ellipse, and $\frac{1}{2} \phi_{\text {ellipse }}$ are polar coordinates on the Poincaré sphere representing the polarization (the eccentricity is $\sqrt{2 \tan (\mu / 2)} /(1+\tan (\mu / 2))$. Thus

$$
w_{\text {circular }}=\tan \frac{1}{2} \mu \exp \left(2 \mathrm{i} \phi_{\text {ellipse }}\right),
$$

so that the index of a $\mathrm{C}$ point is given by the change $\Delta \arg w_{\text {circular }}$ around it, that is, around the corresponding zero of $P_{1}$ or $P_{2}$,

$$
\text { index }=\frac{\Delta \phi_{\text {ellipse }}}{2 \pi}=\frac{\Delta \arg w_{\text {circular }}}{4 \pi} .
$$

Another consequence of (4.12) is that the contours of constant ellipse orientationthe isogyres - are visible as lines of constant hue in figure 3.

When there is chirality, that is, when $G \neq 0$, it follows from (3.21) that the singularities of $\arg w$ are zeros where $P_{1}$ vanishes, so $w \sim Z$, and poles where $P_{2}$ vanishes, so $w \sim 1 / Z^{*}$. Around both types of point, $\Delta \arg w_{\text {circular }}=2 \pi$, so all $\mathrm{C}$ points have index $+\frac{1}{2}$, corresponding to a half rotation of each of the two polarization ellipses (figure $4 a$ ). The total index on the whole $\boldsymbol{s}$ sphere, for each of the states $\boldsymbol{d}^{ \pm}$, is $4 \times\left(+\frac{1}{2}\right)=+2$, as guaranteed by the Euler-Poincaré theorem about the singularities of smooth fields on manifolds.

When there is no chirality (i.e. $G=0$ ), and there is dichroism, the C points coincide with the singular axes. Equation (3.21) shows that $w_{\text {circular }}^{ \pm}= \pm \sqrt{P_{1} / P_{2}}$, so that the zeros are now square-root branch points, around which $\Delta \arg w_{\text {circular }}=\pi$. Equation (4.13) now implies that each of the $\mathrm{C}$ points has index $+\frac{1}{4}$. In this situation, unprecedented in polarization optics, each polarization ellipse describes a quarter rotation around a circuit of each branch point (figure $4 b$ ). The pattern of ellipse pairs on the two sheets connected at a singular axis is, however, single valued, because the circuit connects each ellipse smoothly with its partner (figure $4 b$ ). Since there are eight singular axes, the total index of the $\mathrm{C}$ points on the $\boldsymbol{s}$ sphere is again +2 , as it must be.

Proc. R. Soc. Lond. A (2003) 
(a)

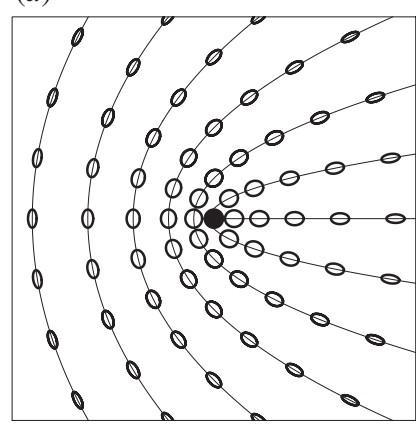

(b)

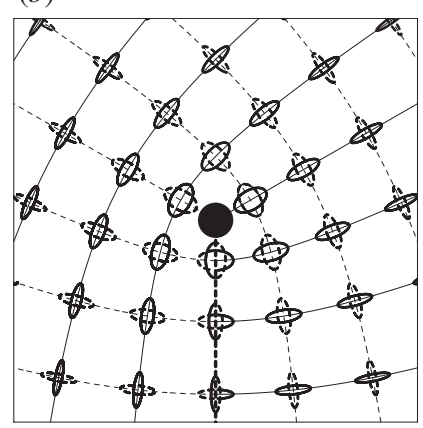

(c)

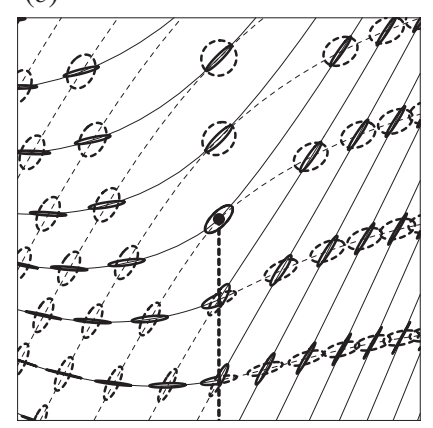

Figure 4. Polarization ellipses near different types of singularity. $(a)$ Index $+\frac{1}{2}$, near a $\mathrm{C}$ point of an absorbing chiral crystal; on a circuit of the $\mathrm{C}$ point, each set of ellipse axes returns after a half-rotation. (b) Index $+\frac{1}{4}$, near a $\mathrm{C}$ point of an absorbing non-chiral crystal; on a circuit of the $\mathrm{C}$ point, the pair of crossed ellipse axes returns after a quarter-rotation. $(c)$ Index 0 , near a singular axis of an absorbing chiral crystal; the axes of each set of ellipses jump across the cut, but the jump vanishes as the singular axis is approached, and the axes of the non-orthogonal pair of ellipses is smooth on the two-sheeted surface.

Consider now a given polarization, for example, $\boldsymbol{d}^{+}$. As $G$ varies smoothly through zero, each of the index $+\frac{1}{2} \mathrm{C}$ points is approached by one of a pair of singular axes, and then jumps to the other singular axis before this recedes. Thus $G=0$ is a transitional case, where the index is shared between two singular axes. For $\boldsymbol{d}^{-}$, the jumps are reversed. Alternatively, we can regard each $\mathrm{C}$ point as fixed (according to the haunting theorem) but transferring its allegiance from one sheet to the other as the singular axis passes through it. The singular axes for $G \neq 0$ possess zero index (they are not $\mathrm{C}$ points), because the state of polarization at such a point is not circular, so the axes of the polarization ellipse are well defined (figure $4 c$ ).

For a transparent non-chiral crystal, equation (3.21) shows that each $\mathrm{C}$ point, now coinciding with an optic axis, again has index $+\frac{1}{2}$ (Berry et al. 1999), i.e. $w_{\text {circular }}^{ \pm}=$ $\pm P_{1}(Z) /\left|P_{1}(Z)\right|$. In the degenerate case of a uniaxial transparent non-chiral crystal, the optic axes, and therefore the $\mathrm{C}$ points, coalesce in antipodal pairs (the same for the two polarizations), each of which has index +1 .

\section{(c) L (linear polarization) lines}

The condition for polarization to be purely linear is

$$
\operatorname{Im} \boldsymbol{d}_{\text {stereographic }}^{*} \times \boldsymbol{d}_{\text {stereographic }}=2\left(\left|d_{\mathrm{R}}\right|^{2}-\left|d_{\mathrm{L}}\right|^{2}\right)=0 \quad \Rightarrow \quad\left|w_{\text {circular }}\right|=1 .
$$

This is a single real equation, so linear polarization is codimension 1, corresponding to lines on the $\boldsymbol{s}$ sphere or the $\boldsymbol{R}$-plane; these are the L lines (Nye $1983 b$ (who called them S lines), 1999; Berry 2001; Dennis 2002).

Equation (3.21) now gives alternative forms for the equations determining the L lines of $\boldsymbol{d}^{ \pm}$,

$$
\left|P_{1}\right|^{2}=\left| \pm \sqrt{P_{1} P_{2}+G^{2}}+G\right|^{2}, \quad\left|P_{2}\right|^{2}=\left| \pm \sqrt{P_{1} P_{2}+G^{2}}-G\right|^{2} .
$$

Proc. R. Soc. Lond. A (2003) 

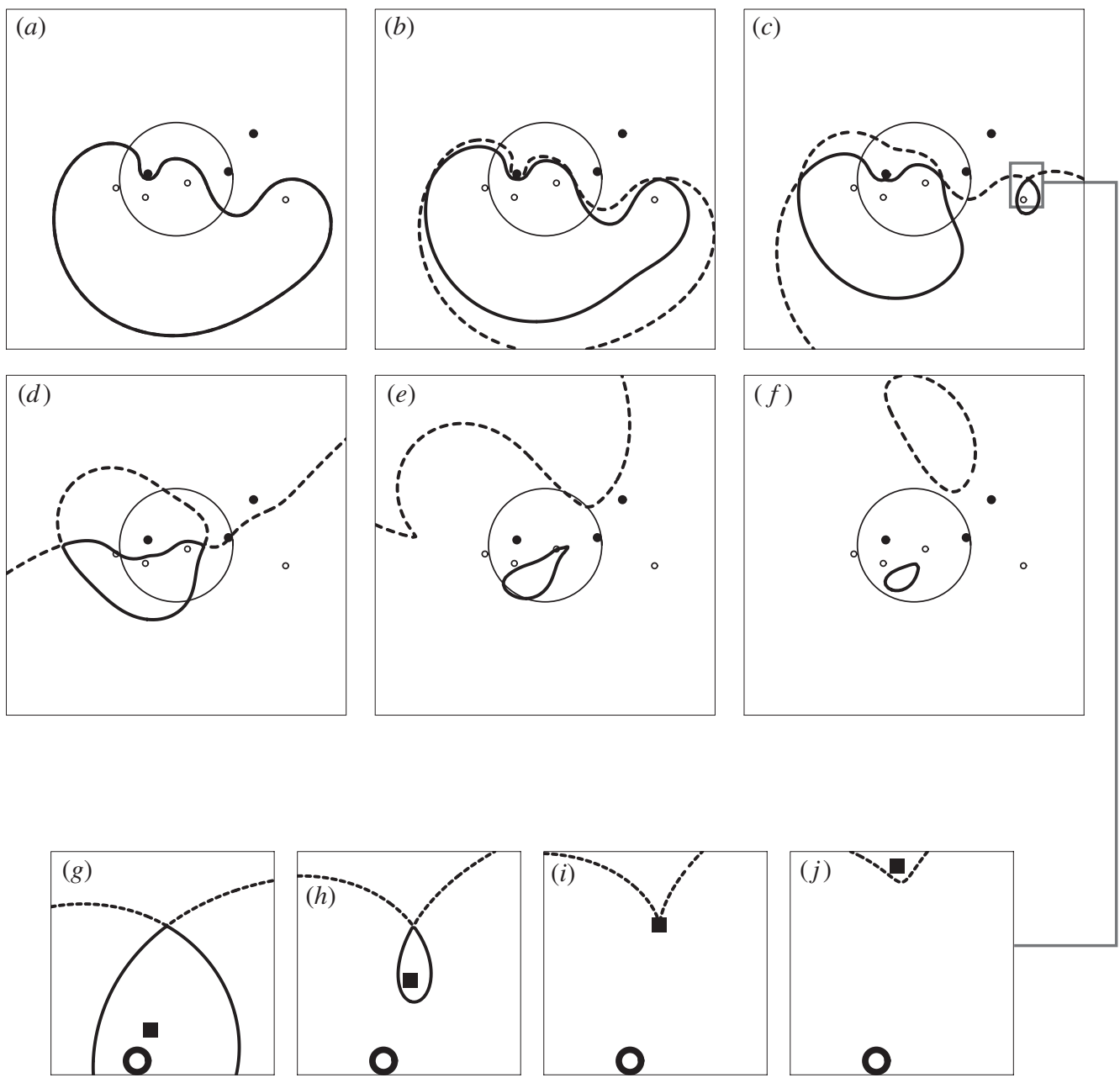

Figure 5. L lines $\boldsymbol{d}^{+}$(full) and $\boldsymbol{d}^{-}$(dashed) in the $\boldsymbol{R}$-plane $|X|<3,|Y|<3$, for the crystal (2.7). (a) $A=0.1, \Gamma=0$; (b) $A=0.1, \Gamma=0.00025$; (c) $A=0.1, \Gamma=0.0008$; $(d) A=0.1$, $\Gamma=0.0025$; (e) $A=0.1, \Gamma=0.0035 ;(f) A=0.1, \Gamma=0.0038$, and magnifications in the range $1.7<X<2.3,-0.4<Y<0.2$ for $(g) A=0.1, \Gamma=0.0008$; $(h) A=0.1$, $\Gamma=0.0012 ;(i) A=0.1, \Gamma=0.00145 ;(j) A=0.1, \Gamma=0.0016$. Filled/open circles denote the right-/left-handed $\mathrm{C}$ points of $\boldsymbol{d}^{+} / \boldsymbol{d}^{-}$. In $(g)-(j)$, the filled squares denote the singular axes, migrating away from the $\mathrm{C}$ points (which remain fixed according to the haunting theorem); the L loop disappears in $(i)$ as the singular axis crosses the optic axis. Parts $(a)-(f)$ illustrate the antipodal relation (see $\S 5$ ) between the + and - L lines in the northern hemisphere (inside the indicated unit circle) and the southern hemisphere.

Adding these equations gives, after some manipulation,

$$
\left|P_{1}\right|^{2}-\left|P_{2}\right|^{2}=2 \varepsilon\left|G^{*} P_{1}+G P_{2}^{*}\right|, \quad \varepsilon= \pm 1
$$

(the minus sign on the left-hand side is not an error). Squaring this equation specifies the union of the L lines for $\boldsymbol{d}^{+}$and $\boldsymbol{d}^{-}$. To determine the sign $\varepsilon$, and thereby the parts of the $\mathrm{L}$ lines associated with $\boldsymbol{d}^{+}$and $\boldsymbol{d}^{-}$separately, we subtract the two equations 
in (4.15), leading to

$$
\varepsilon= \pm \operatorname{sgn} \operatorname{Re} G^{*} \sqrt{P_{1} P_{2}+G^{2}} .
$$

The L lines separate the $\boldsymbol{s}$ sphere or $\boldsymbol{R}$-plane into regions of right- and left-handed polarization.

Figure 3 shows L lines in the northern hemisphere, and figure 5 shows global views illustrating the antipodal connections to be discussed in $\S 5$. The L lines snake around the $\boldsymbol{R}$-plane, avoiding the $\mathrm{C}$ points. When there is absorption but no chirality, the L lines for $\boldsymbol{d}^{+}$and $\boldsymbol{d}^{-}$are the same (parts $(b+)$ and $(b-)$ of figure 3 and figure $5 a$ ). For chiral crystals, the + and $-\mathrm{L}$ lines are different (parts $(c)-(f)$ of figure 3 and parts $(b)-(f)$ of figure 5$)$. Across a branch cut, the L lines for each state are discontinuous but match smoothly onto each other (parts $(e+)$ and $(e-)$ of figure 3 and parts $(d),(e),(h)$ of figure 5$)$.

When $G$ is real, that is, without circular dichroism, equations (3.13), (3.14) and (4.6) imply that in the absorption-dominated regime the L lines, satisfying (4.15), cross at the optic axes defined by (4.2); parts $(b)-(d)$ of figure 5 show several examples. Crossing L lines can form loops (parts $(c),(d),(g),(h)$ of figure 5$)$. An L loop can shrink and disappear as a singular axis crosses an L line, corresponding to one of the critical values of chirality in (4.7) (parts $(g)-(j)$ of figure 5$)$. In the chiral-dominated regime, there are no L lines; figure $5 f$ shows an approach to this situation, in which the lines have shrunk to two small antipodal loops.

\section{Antipodal symmetries}

If the direction of the light is reversed, that is, $s \rightarrow-s$, Maxwell's equation (2.8), together with the formulae (2.3) and (2.5) for the dielectric tensor, imply that the electric displacement $\boldsymbol{D}$, regarded as a 3 -vector, is unaltered, provided the chirality is reversed too,

$$
\boldsymbol{D}(\boldsymbol{s}, \boldsymbol{u}, \boldsymbol{\gamma})=\boldsymbol{D}(-\boldsymbol{s}, \boldsymbol{u},-\gamma)
$$

An immediate implication of this antipodal transformation can be written for the ratio of components in the polar representation $\boldsymbol{d}_{\text {polar }}$ (equation (3.1)), because the component $d_{\theta}$ is antipodally invariant, while the component $d_{\phi}$ changes sign,

$$
w_{\text {polar }}^{ \pm}\left(\frac{1}{Z^{* 2}}, G\right)=-w_{\text {polar }}^{ \pm}(z,-G) .
$$

To explore the implications of (5.1) in other representations, we first note that under the antipodal transformation (3.4) the polynomials (3.13)-(3.16) transform as

$$
\left.\begin{array}{ll}
P_{1}\left(-\frac{1}{Z^{*}}\right)=\frac{1}{Z^{* 4}} P_{2}(Z), & G\left(-\frac{\boldsymbol{R}}{R^{2}}\right)=\frac{1}{R^{4}} G(\boldsymbol{R}), \\
P_{2}\left(-\frac{1}{Z^{*}}\right)=\frac{1}{Z^{4}} P_{1}(Z), & Q\left(-\frac{\boldsymbol{R}}{R^{2}}\right)=\frac{1}{R^{4}} Q(\boldsymbol{R}) .
\end{array}\right\}
$$

This implies that the eigenvalues (3.17) are antipodally invariant, even if the chirality $G$ is not reversed,

$$
\lambda^{ \pm}\left(-\frac{1}{Z^{*}}, G\right)=\lambda^{ \pm}(Z, G) .
$$

Proc. R. Soc. Lond. A (2003) 


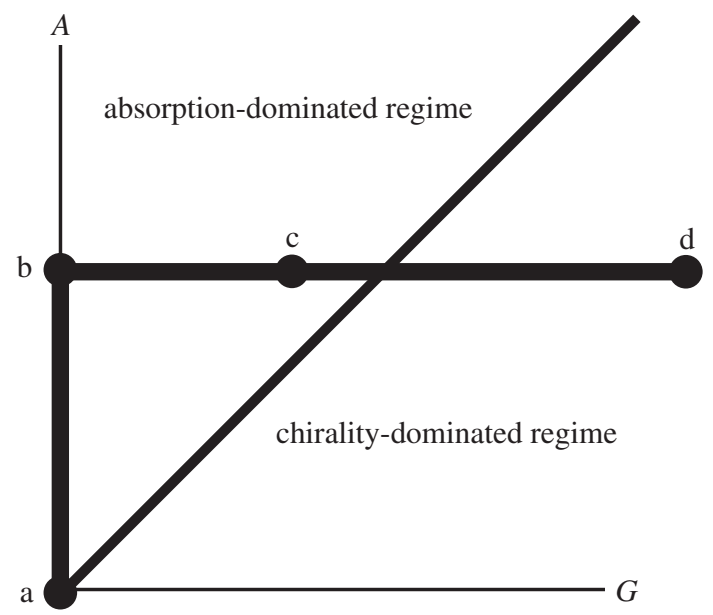

Figure 6. Parameter plane for the local model of $\S 6$, showing the absorption- and chiralitydominated regimes and the path abcd in crystal space illustrated in figures 7 and 8 .

The polarizations $\boldsymbol{d}^{ \pm}$are not antipodally invariant. From (5.2) or (5.3), it follows that the ratio of components (3.21) in the circular basis transforms as

$$
w_{\text {circular }}^{ \pm}\left(-\frac{1}{Z^{*}}, G\right)=\frac{\exp (4 \mathrm{i} \phi)}{w_{\text {circular }}^{ \pm}(Z,-G)} .
$$

A consequence of this relation is

$$
\left|w_{\text {circular }}^{ \pm}\left(-\frac{1}{Z^{*}}, G\right)\right|=\frac{1}{\left|w_{\text {circular }}^{ \pm}(Z,-G)\right|}=\frac{1}{\left|w_{\text {circular }}^{\mp}(Z, G)\right|} .
$$

This explains the symmetry of the L lines in parts $(a)-(f)$ of figure 5 : lines for $\boldsymbol{d}^{+}$ inside the unit circle are related to lines for $\boldsymbol{d}^{-}$outside the circle, and vice versa. Finally, the ratio (3.24) in stereographic coordinates transforms as

$$
w_{\text {stereographic }}^{ \pm}\left(-\frac{1}{Z^{*}}, G\right)=\frac{\sin 2 \phi-\cos 2 \phi w_{\text {stereographic }}^{ \pm}(z,-G)}{\cos 2 \phi+\sin 2 \phi w_{\text {stereographic }}^{ \pm}(z,-G)} .
$$

\section{Local model}

To study the singularities described in $\S 4$ in more detail, we consider a local model that captures much of the physics. The model incorporates one pair of singular axes, so each of the principal polynomials $P_{1}$ and $P_{2}$ possesses a single zero. The polynomial $G$ is chosen to be a real constant, and the irrelevant polynomial $Q$ is set equal to zero. Thus we replace (3.13)-(3.16) by

$$
P_{1}(Z)=Z+\mathrm{i} A, \quad P_{2}(Z)=Z^{*}+\mathrm{i} A, \quad G(\boldsymbol{R})=G, \quad Q=0 .
$$

The real constant $A$ describes absorption, and the constant $G$ describes chirality, so crystal space is two dimensional (figure 6) (as in (2.7)). In the matrix (3.12), the factor $2\left(1+R^{2}\right)^{2}$ is unimportant, so polarizations are determined by

$$
\boldsymbol{m}_{\text {circular }}(\boldsymbol{R})=\left(\begin{array}{cc}
G & X-\mathrm{i} Y+\mathrm{i} A \\
X+\mathrm{i} Y+\mathrm{i} A & -G
\end{array}\right) .
$$

Proc. R. Soc. Lond. A (2003) 

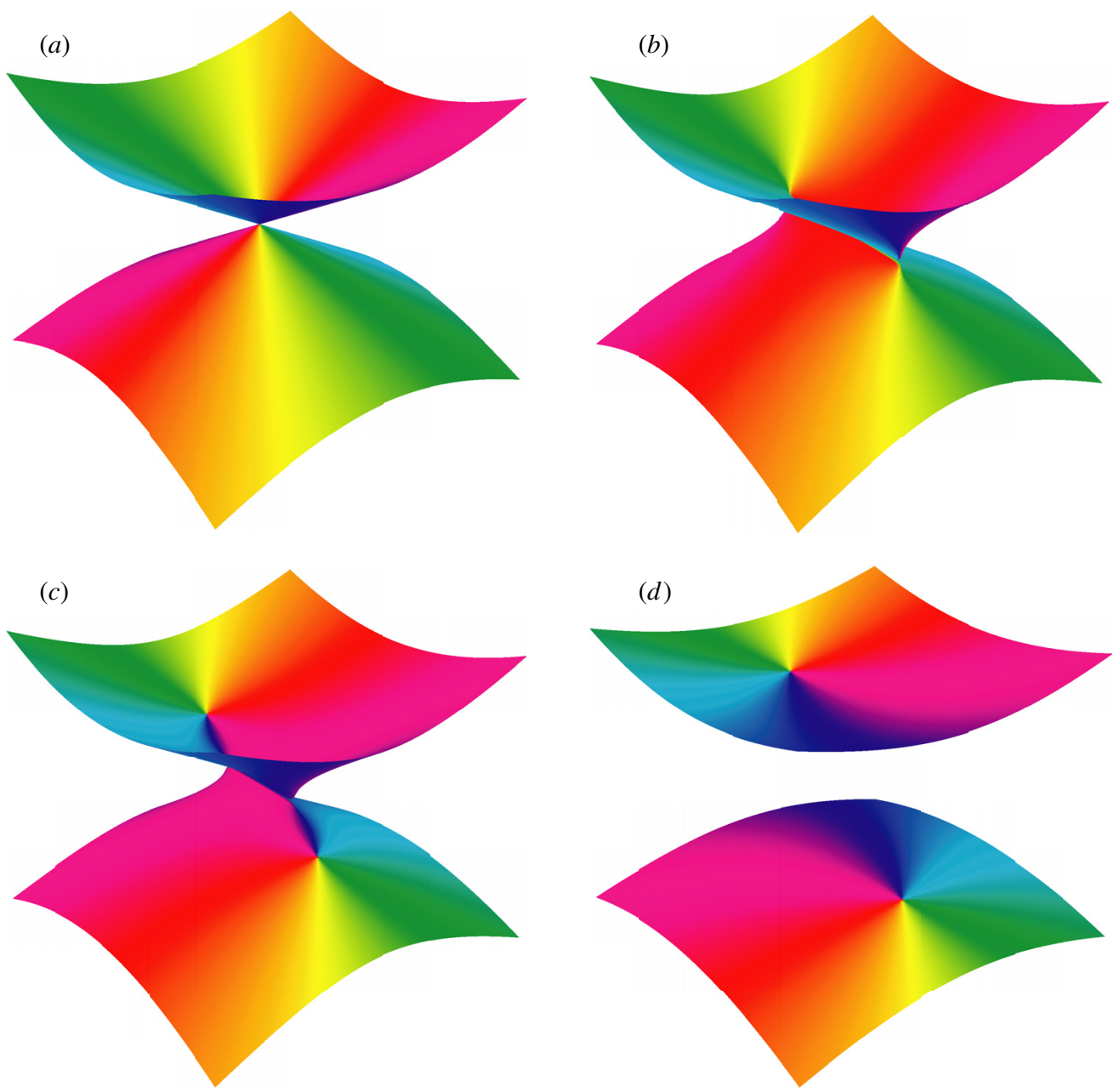

Figure 7. Eigenvalue sheets $\operatorname{Re} \lambda^{ \pm}$for the local model (6.1), computed from (6.3), with $\arg w^{ \pm}$ colour coded by hue, for $|X|<2,|Y|<2$ : (a) $A=0, \Gamma=0$ (transparent non-chiral); (b) $A=1$, $\Gamma=0$ (absorbing non-chiral); $(c) A=1, \Gamma=0.8$ (absorption dominated); $(d) A=0, \Gamma=2$ (chirality dominated).

The eigenvalues are

$$
\lambda^{ \pm}(\boldsymbol{R})= \pm \sqrt{R^{2}+G^{2}-A^{2}+2 \mathrm{i} A X}
$$

and are displayed in figure 7 as sheets above the $\boldsymbol{R}$-plane, for crystals along the path in figure 6 . The degeneracies (see $\S 4 a$ ) are at

$$
X=0, \quad Y= \pm \sqrt{A^{2}-G^{2}} .
$$

In this model there is a single optic axis, at $\boldsymbol{R}=\mathbf{0}$ (figure $7 a$ ). For $|A|>|G|$ (absorption-dominated regime), the optic axis splits into the two singular axes. The two sheets $\lambda^{+}$and $\lambda^{-}$are connected by a cut, which, according to the branch specification (3.18), is a straight line joining the singular axes (parts (b) and (c) of figure 7).

Proc. R. Soc. Lond. A (2003) 

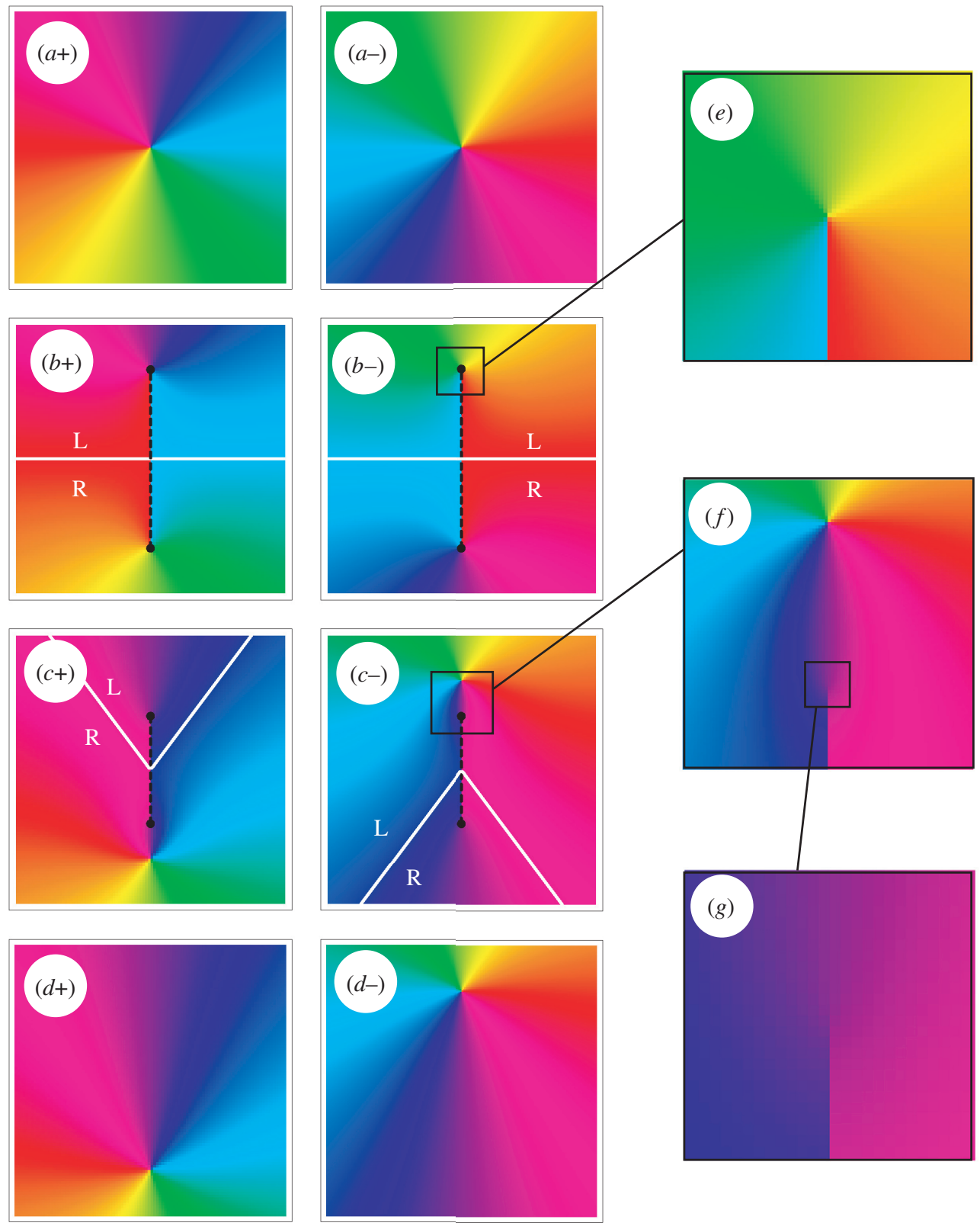

$\mathrm{L}$

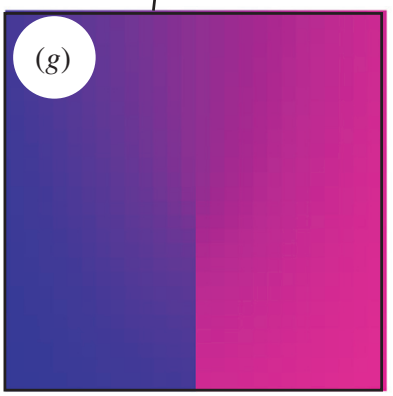

Figure 8. $(a+)-(d+) \arg w^{+},(a-)-(d-)$ : $\arg w^{-}$, for the local model (6.1), colour coded by hue, for $|X|<2,|Y|<2$ and $A, \Gamma$ as in parts $(a)-(d)$ of figure 7. Black dots denote singular axes, connected by branch cuts (dashed lines), and white lines denote L lines. (e) Magnification of $(b-)$, for $|X|<0.25,0.75<Y<1.25$, showing the index $\frac{1}{4}$ singularity at the singular axis. ( $f$ ) Magnification of $(c-)$, for $|X|<0.35,0.4<Y<1.1$, showing the index $\frac{1}{2}$ singularity at the $\mathrm{C}$ point (above) and the index-zero singularity at the singular axis (below). ( $g$ ) Magnification of $(f)$, for $|X|<0.05,0.55<Y<0.65$, showing how the jump in $\arg w$ fades away as the singular axis is approached. 
These axes coalesce, again at $\boldsymbol{R}=\mathbf{0}$, when $|A|=|G|$, corresponding to the chiralityabsorption boundary, as in (4.7). For $|G|>|A|$, in the chirality-dominated regime, the sheets have separated (figure $7 d$ ). (In this model, with only one pair of singular axes, the chirality-absorption boundary is sharp.)

The $\mathrm{C}$ points (see $\S 4 b$ ) are at the zeros of $P_{1}$ and $P_{2}$, namely,

$$
\left.\begin{array}{lll}
X=0, \quad Y=A & \Rightarrow & \text { C point of } \boldsymbol{d}^{-}, \mathrm{L} \text { type }\left(d_{\mathrm{R}}=0\right), \\
X=0, \quad Y=-A & \Rightarrow & \text { C point of } \boldsymbol{d}^{+}, \mathrm{R} \text { type }\left(d_{\mathrm{L}}=0\right) .
\end{array}\right\}
$$

As expected from the haunting theorem, these positions are independent of $G$, and remain fixed at the positions of the singular axes of the non-chiral absorbing crystal, as can be seen clearly in parts $(b)-(d)$ of figure 7 and parts $(b)-(d)$ of figure 8 .

The L lines (see $\S 4 c$ ) are determined by equations (4.16) and (4.17), which give the explicit solution

$$
Y=+\frac{G|X|}{\sqrt{A^{2}-G^{2}}} \quad\left(\text { for } \boldsymbol{d}^{+}\right), \quad Y=-\frac{G|X|}{\sqrt{A^{2}-G^{2}}} \quad\left(\text { for } \boldsymbol{d}^{-}\right) .
$$

These divide the $\boldsymbol{R}$-plane into $\mathrm{V}$-shaped regions (parts $(c),(d)$ of figure 8), pointing down for $\boldsymbol{d}^{+}$and up for $\boldsymbol{d}^{-}$; in both cases, the regions below/above the $\mathrm{L}$ lines have right-/left-handed polarization. It can be shown that in the local model the L lines are also isogyres, that is, contours of $\arg w$, visible as lines of constant hue in figure $8 c$.

The magnifications in parts $(e)-(g)$ of figure 8 illustrate the different polarization singularities shown in figure 4 . The index $+\frac{1}{4}$ singularity of figure $4 b$, for an absorbing non-chiral crystal, is shown in figure $8 e$; around the $\mathrm{C}$ point, which here is also a singular axis, the phase (indicated by hue) increases by $\pi$, so the ellipse axis rotates by $\frac{1}{2} \pi$ (cf. equation (4.13)). The index $+\frac{1}{2}$ singularity of figure $4 a$, for an absorbing chiral crystal, at a $\mathrm{C}$ point detached from a singular axis, is shown in figure $8 f$; around the $\mathrm{C}$ point, the phase increases by $2 \pi$, so the ellipse axis rotates by $\pi$. The index-zero singularity of figure $4 c$ at a singular axis, where the phase discontinuity on each sheet across the cut fades away as the singular axis is approached, is shown in figure $8 g$.

It can be verified that making the chirality parameter $G$ complex, corresponding to circular dichroism, does not lead to essential changes in the deportment of polarization singularities just described. The only difference is that the $\mathrm{L}$ lines no longer cross at the optic axes: if $G=G_{1}+\mathrm{i} G_{2}$, then, in (6.6), $G$ is replaced by $G_{1}$ and $X$ by $X+A G_{2} / G_{1}$.

\section{Interference figures}

In this section we consider the arrangement, familiar in crystal optics (Ramachandran \& Ramaseshan 1961), of a divergent beam of light incident on a crystal slab, with or without a polarizer or analyser, and the emerging light viewed on a distant screen or with a microscope. Each point on the screen then corresponds to light travelling in a particular direction $\boldsymbol{R}$.

After the light traverses a slab of thickness $L$, each of the polarizations acquires an exponential factor,

$$
\boldsymbol{d}^{ \pm} \rightarrow \boldsymbol{d}^{ \pm} \exp \left\{\mathrm{i}\left(\bar{\sigma} \pm \frac{1}{2} \Delta \sigma\right)\right\}
$$

Proc. R. Soc. Lond. A (2003) 
where

$$
\bar{\sigma}(\boldsymbol{R})=k L \frac{\left(1+R^{2}\right)}{\left(1-R^{2}\right)} \bar{n}(\boldsymbol{R}), \quad \Delta \sigma(\boldsymbol{R})=k L \frac{\left(1+R^{2}\right)}{\left(1-R^{2}\right)} \Delta n(\boldsymbol{R}),
$$

incorporating the mean and difference of the two refractive indices defined in (3.19) and the slant distance travelled by the light (the normal to the slab being defined as the $z$-axis). For a transparent crystal, the exponentials in (7.1) are phase factors; when there is absorption, each exponent has a real part. (The familiar quarter- and half-wave plates are very special cases, in which the crystal is transparent, non-chiral and uniaxial, with the axis parallel to the slab, which is illuminated normally, and $\Delta \sigma=\frac{1}{2} \pi$ or $\pi$.)

The ways in which these exponential factors manifest themselves depend on whether and how the incident light is polarized and whether the light emerging from the crystal is viewed through an analyser. We discuss the four different cases separately; the analysis relies on several matrix relations, listed in the appendix. In particular, the invariance of all physical quantities in the formulae of this section under unitary change of basis for $\boldsymbol{d}$ is guaranteed by the two relations (A 9) and (A 11); therefore, in what follows, it is not necessary to specify the basis.

Our aim in this section is to provide useful general formulae, and to show how the singularities we have been discussing can be exhibited in interference figures. For the latter purpose, we use only the crossed polarizer/analyser arrangement, which gives the clearest fringes.

\section{(a) Polarized incident light, no analyser}

Consider light entering the crystal after being polarized in the state $\boldsymbol{d}_{0}$. Resolution of $\boldsymbol{d}_{0}$ into a superposition of the two polarizations corresponding to the direction $\boldsymbol{s}$, according to (A 4), and use of (A 5), and then propagating each polarization according to (7.1), leads to the following formula for the light vector emerging from the crystal:

$$
\boldsymbol{d}_{0} \rightarrow \boldsymbol{d}_{\text {final }}(\boldsymbol{R})=\frac{\exp (\mathrm{i} \bar{\sigma})}{\overline{\boldsymbol{d}}^{+} \cdot \boldsymbol{d}^{+}}\left[\overline{\boldsymbol{d}}^{+} \cdot \boldsymbol{d}_{0} \exp \left(\frac{1}{2} \mathrm{i} \Delta \sigma\right) \boldsymbol{d}^{+}-\overline{\boldsymbol{d}}^{-} \cdot \boldsymbol{d}_{0} \exp \left(-\frac{1}{2} \mathrm{i} \Delta \sigma\right) \boldsymbol{d}^{-}\right] .
$$

Here, the overbars denote left eigenvectors as defined in (A 1). The intensity $I$ of this light, if observed directly (i.e. without an analyser) is

$$
\begin{aligned}
& I=\boldsymbol{d}_{\text {final }}^{*} \cdot \boldsymbol{d}_{\text {final }} \\
& =\frac{\exp (-2 \operatorname{Im} \bar{\sigma})}{\left|\overline{\boldsymbol{d}}^{+} \cdot \boldsymbol{d}^{+}\right|^{2}} \\
& \quad \times\left[\left|\overline{\boldsymbol{d}}^{+} \cdot \boldsymbol{d}_{0}\right|^{2} \exp (-\operatorname{Im} \Delta \sigma)+\left|\overline{\boldsymbol{d}}^{-} \cdot \boldsymbol{d}_{0}\right|^{2} \exp (\operatorname{Im} \Delta \sigma)\right. \\
& \left.\quad \quad \quad-2 \operatorname{Re}\left(\overline{\boldsymbol{d}}^{-*} \cdot \boldsymbol{d}_{0}^{*}\right)\left(\overline{\boldsymbol{d}}^{+} \cdot \boldsymbol{d}_{0}\right)\left(\boldsymbol{d}^{+} \cdot \boldsymbol{d}^{-*}\right) \exp (\mathrm{i} \operatorname{Re} \Delta \sigma)\right] .
\end{aligned}
$$

The term involving $\operatorname{Re} \Delta \sigma$ represents interference between the two polarizations. If the crystal is transparent, this term vanishes, because $\boldsymbol{d}^{+} \cdot \boldsymbol{d}^{-*}=0$ by the complex orthogonality of eigenvectors of Hermitian matrices. As is well known (Ramachandran \& Ramaseshan 1961), it is only for absorbing crystals that interference can be observed with this arrangement.

Proc. R. Soc. Lond. A (2003) 


\section{(b) Unpolarized incident light, no analyser}

Unpolarized incident light (Ramachandran \& Ramaseshan 1961; Brosseau 1998) can be represented by averaging the intensity (7.4) over any pair of orthogonal incident polarizations $\boldsymbol{d}_{0}$, for example,

$$
\boldsymbol{d}_{01}=\left(\begin{array}{l}
1 \\
0
\end{array}\right) \quad \text { and } \quad \boldsymbol{d}_{02}=\left(\begin{array}{l}
0 \\
1
\end{array}\right)
$$

Thus, averaging corresponds to the operation

$$
\left\langle f\left(\boldsymbol{d}_{0}\right)\right\rangle=\frac{1}{2}\left[f\left(\boldsymbol{d}_{01}\right)+f\left(\boldsymbol{d}_{02}\right)\right]
$$

and leads to

$$
\left\langle\left|\boldsymbol{a} \cdot \boldsymbol{d}_{0}\right|^{2}\right\rangle=\frac{1}{2}, \quad\left\langle\boldsymbol{a} \cdot \boldsymbol{d}_{0} \boldsymbol{b} \cdot \boldsymbol{d}_{0}^{*}\right\rangle=\frac{1}{2} \boldsymbol{a} \cdot \boldsymbol{b}
$$

for any normalized vectors $\boldsymbol{a}, \boldsymbol{b}$. (Averaging over all vectors $\boldsymbol{d}_{0}$ by integration over the Poincaré sphere of polarizations gives the same result.)

Thus the intensity (7.4) becomes

$$
I=\left\langle\boldsymbol{d}_{\text {final }}^{*} \cdot \boldsymbol{d}_{\text {final }}\right\rangle=\frac{\exp (-2 \operatorname{Im} \bar{\sigma})}{\left|\overline{\boldsymbol{d}}^{+} \cdot \boldsymbol{d}^{+}\right|^{2}}\left[\cosh (\operatorname{Im} \Delta \sigma)-\left|\boldsymbol{d}^{+*} \cdot \boldsymbol{d}^{-}\right|^{2} \cos (\operatorname{Re} \Delta \sigma)\right] .
$$

Again, the interference term vanishes for a transparent crystal.

The two scalar products are related by (A 7), and calculations based on (3.20) and (3.21) lead to the explicit expression

$$
\left|\overline{\boldsymbol{d}}^{+} \cdot \boldsymbol{d}^{+}\right|^{2}=\frac{\left|w^{+}-w^{-}\right|^{2}}{\left(1+\left|w^{+}\right|^{2}\right)\left(1+\left|w^{-}\right|^{2}\right)}=\frac{4\left|P_{1} P_{2}+G^{2}\right|}{\left|P_{1}\right|^{2}+\left|P_{2}\right|^{2}+2\left(\left|P_{1} P_{2}+G^{2}\right|+|G|^{2}\right)} .
$$

\section{(c) Unpolarized incident light, analyser}

In this case, the light (7.3) is analysed by projection onto a state $\boldsymbol{d}_{1}$, and then the intensity is averaged over all $\boldsymbol{d}_{0}$ as in $\S 7 b$. Use of the averages (7.7) leads to

$$
\begin{aligned}
& I=\left\langle\left|\boldsymbol{d}_{\text {final }} \cdot \boldsymbol{d}_{1}^{*}\right|^{2}\right\rangle_{\boldsymbol{d}_{0}} \\
& =\frac{\exp (-2 \operatorname{Im} \bar{\sigma})}{2\left|\overline{\boldsymbol{d}}^{+} \cdot \boldsymbol{d}^{+}\right|^{2}} \\
& \quad \times\left[\left|\boldsymbol{d}^{+} \cdot \boldsymbol{d}_{1}^{*}\right|^{2} \exp (-\operatorname{Im} \Delta \sigma)+\left|\boldsymbol{d}^{-} \cdot \boldsymbol{d}_{1}^{*}\right|^{2} \exp (\operatorname{Im} \Delta \sigma)\right. \\
& \left.\quad \quad-2 \operatorname{Re}\left(\boldsymbol{d}^{-*} \cdot \boldsymbol{d}_{1}\right)\left(\boldsymbol{d}^{+} \cdot \boldsymbol{d}_{1}^{*}\right)\left(\boldsymbol{d}^{-} \cdot \boldsymbol{d}^{+*}\right) \exp (\mathrm{i} \operatorname{Re} \Delta \sigma)\right] .
\end{aligned}
$$

This closely resembles the result (7.4), corresponding to a polarizer but no analyser. Indeed, the replacements

$$
\boldsymbol{d}^{ \pm} \rightarrow \overline{\boldsymbol{d}}^{ \pm}, \quad \boldsymbol{d}_{1} \rightarrow \boldsymbol{d}_{0}^{*}
$$

in (7.10) reproduce (7.4) exactly (apart from a trivial factor $\frac{1}{2}$ describing the unpolarized incident light in (7.10)). (This polarizer-analyser exchange is illustrated experimentally in fig. 71 of Ramachandran \& Ramaseshan (1961).)

Proc. R. Soc. Lond. A (2003) 
(d) Polarizer and analyser

In this arrangement, the light (7.3) emerging after traversing the crystal is observed after projection onto a state $\boldsymbol{d}_{1}$, so the intensity is

$$
\begin{aligned}
I= & \left|\boldsymbol{d}_{\text {final }} \cdot \boldsymbol{d}_{1}^{*}\right|^{2} \\
= & \frac{\exp (-2 \operatorname{Im} \bar{\sigma})}{\left|\overline{\boldsymbol{d}}^{+} \cdot \boldsymbol{d}^{+}\right|^{2}} \\
& \quad \times\left|\left(\overline{\boldsymbol{d}}^{+} \cdot \boldsymbol{d}_{0}\right)\left(\boldsymbol{d}^{+} \cdot \boldsymbol{d}_{1}^{*}\right) \exp \left(\frac{1}{2} \mathrm{i} \Delta \sigma\right)-\left(\overline{\boldsymbol{d}}^{-} \cdot \boldsymbol{d}_{0}\right)\left(\boldsymbol{d}^{-} \cdot \boldsymbol{d}_{1}^{*}\right) \exp \left(-\frac{1}{2} \mathrm{i} \Delta \sigma\right)\right|^{2} \\
= & \frac{\exp (-2 \operatorname{Im} \bar{\sigma})}{\left|w^{+}-w^{-}\right|^{2}\left(1+\left|w_{0}\right|^{2}\right)\left(1+\left|w_{1}^{a}\right|^{2}\right)} \\
& \quad \times\left|\left(w^{-}-w_{0}\right)\left(w^{+}-w_{1}^{a}\right) \exp \left(\frac{1}{2} \mathrm{i} \Delta \sigma\right)-\left(w^{+}-w_{0}\right)\left(w^{-}-w_{1}^{a}\right) \exp \left(-\frac{1}{2} \mathrm{i} \Delta \sigma\right)\right|^{2},
\end{aligned}
$$

where in the second equality the ratios $w$ (e.g. (3.21)) have been used, and the symmetrical form obtained by writing $w_{1}$ in terms of the antipodal (orthogonal) state on the Poincaré sphere, namely,

$$
w_{1}^{a}=-\frac{1}{w_{1}^{*}} .
$$

Note that the expression (7.12) is symmetric under exchange of polarizer and analyser and + and - states, in the form $w^{+} \leftrightarrow w^{-}, w_{0} \leftrightarrow w_{1}^{a}$.

In the common situation of crossed polarizer and analyser, that is, $\boldsymbol{d}_{1}^{*} \cdot \boldsymbol{d}_{0}=0$, we can choose

$$
\boldsymbol{d}_{1}=\exp (\mathrm{i} \delta)\left(\begin{array}{c}
-d_{0 \mathrm{~L}}^{*} \\
d_{0 \mathrm{R}}^{*}
\end{array}\right)
$$

After some simplification using (A 2), the intensity (7.12) becomes

$$
\begin{aligned}
I & =\left|\boldsymbol{d}_{\text {final }} \cdot \boldsymbol{d}_{1}^{*}\right|^{2} \\
& =2 \exp (-2 \operatorname{Im} \bar{\sigma}) \frac{\left|\left(\overline{\boldsymbol{d}}^{+} \cdot \boldsymbol{d}_{0}\right)\left(\boldsymbol{d}^{+} \cdot \boldsymbol{d}_{1}^{*}\right)\right|^{2}}{\left|\overline{\boldsymbol{d}}^{+} \cdot \boldsymbol{d}^{+}\right|^{2}}[\cosh (\operatorname{Im} \Delta \sigma)-\cos (\operatorname{Re} \Delta \sigma)] .
\end{aligned}
$$

The combination of scalar products can be expressed explicitly using (3.20) and (3.21), giving

$$
\frac{\left|\left(\overline{\boldsymbol{d}}^{+} \cdot \boldsymbol{d}_{0}\right)\left(\boldsymbol{d}^{+} \cdot \boldsymbol{d}_{1}^{*}\right)\right|^{2}}{\left|\overline{\boldsymbol{d}}^{+} \cdot \boldsymbol{d}^{+}\right|^{2}}=\frac{\left|\left(w_{0}-w^{+}\right)\left(w_{0}-w^{-}\right)\right|^{2}}{\left|\left(w^{+}-w^{-}\right)\right|^{2}\left(1+\left|w_{0}\right|^{2}\right)^{2}}=\frac{\left|d_{0 \mathrm{R}}^{2} P_{1}-d_{0 \mathrm{~L}}^{2} P_{2}+2 d_{0 \mathrm{R}} d_{0 \mathrm{~L}} G\right|^{2}}{4\left|P_{1} P_{2}+G^{2}\right|},
$$

where in the last expression $d_{0 \mathrm{R}}$ and $d_{0 \mathrm{~L}}$ are the components of the initial polarization in the circular basis. In (7.15), the 'cosh-cos' factor describes the interference between the two polarizations, and the prefactor involving the scalar products (7.16) describes the brushes that modulate the interference pattern.

An interesting consequence of (7.15) is that if, for some direction one of the states, $\boldsymbol{d}^{+}$, say, is the same as that of the polarizer $\boldsymbol{d}_{0}$, the prefactor vanishes, so that direction appears dark. The origin of this behaviour can be seen from (7.3), where the coefficient of the state $\boldsymbol{d}^{-}$vanishes (even though $\boldsymbol{d}^{-}$is not orthogonal to $\boldsymbol{d}^{+}$), so that $\boldsymbol{d}^{+}$is the only state that propagates and is extinguished by the analyser. By

Proc. R. Soc. Lond. A (2003) 
(a)

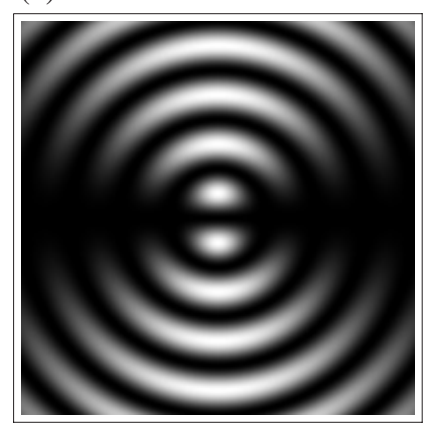

(b)

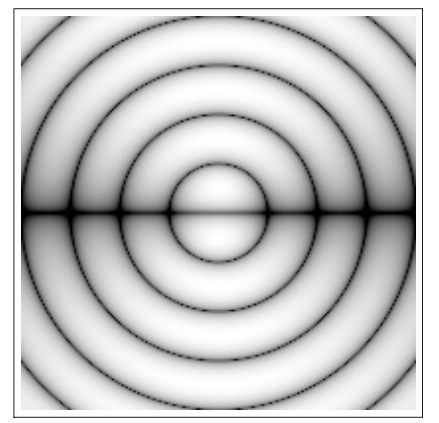

$(c)$

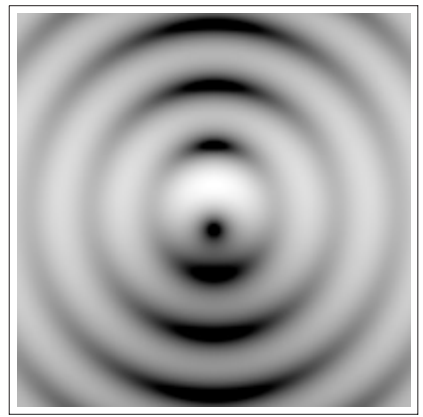

Figure 9. Interference figures for crystal slabs viewed between crossed polarizer and analyser, calculated from (7.15) with the local model of $\S 6$. (a) Density plot of intensity $I$ with crossed linear polarizations, biaxial birefringence only $(A=G=0)$, with $l=5$, for $|X|<5,|Y|<5$. (b) As $(a)$, plotting $\log I$. (c) Density plot of $\log I$ with right circular polarizer and left circular analyser, for a dichroic non-chiral crystal with $A=1, G=0$, with $l=2$, for $|X|<10,|Y|<10$; the black spot corresponds to the lower, right-handed, $\mathrm{C}$ point, which is extinguished by the analyser; the bright spot above it corresponds to the left-handed $\mathrm{C}$ point, whose brightness illustrates the Pancharatnam phenomenon described in the text.

contrast, the state orthogonal to $\boldsymbol{d}_{0}$ is not extinguished - a lack of symmetry between polarizer and analyser that reflects the lack of time-reversal symmetry in this general case (and which is a special case of the general exchange relation stated immediately after (7.13)).

\section{(e) Displaying the polarization singularities}

The simplest and most familiar case of (7.15) and (7.16) is a transparent non-chiral crystal with polarizer and analyser linearly polarized in directions $\gamma, \gamma+\frac{1}{2} \pi$, that is,

$$
G=0, \quad P_{2}=P_{1}^{*}, \quad \bar{\sigma}, \Delta \sigma \text { real, } \quad \boldsymbol{d}_{\text {chiral0 }}=\frac{1}{\sqrt{2}}\left(\begin{array}{c}
\exp (-\mathrm{i} \gamma) \\
\exp (\mathrm{i} \gamma)
\end{array}\right),
$$

for which the formula (7.15) reduces to

$$
I=\left|\boldsymbol{d}_{\text {final }} \cdot \boldsymbol{d}_{1}^{*}\right|^{2}=\frac{\left[\left|P_{1}\right|^{2}-\cos 4 \gamma \operatorname{Re} P_{1}^{2}-\sin 4 \gamma \operatorname{Im} P_{1}^{2}\right]}{2\left|P_{1}\right|^{2}} \sin ^{2} \frac{1}{2} \Delta \sigma .
$$

This was previously derived by Berry et al. (1999) and applied to the interpretation of biaxial conoscopic figures viewed through overhead-projector transparency foil between crossed polarizers. An example, with $I$ calculated with the local model, is shown in figure $9 a$ as a density plot; the interference rings are centred on an optic axis, and the 'bullseye' is crossed by a black brush where the prefactor vanishes. In all subsequent pictures it will be more convenient to plot $\log I$ rather than $I$, and figure $9 b$ shows how this modifies the appearance of the conoscopic figure.

An interesting application of (7.15) is to a dichroic non-chiral crystal viewed between crossed circular polarizers in directions near a pair of singular axes, which are $\mathrm{C}$ points of opposite handedness (see $\S 4 b$ ). One of the axes will have handedness opposite to that of $\boldsymbol{d}_{0}$, and the question arises of how the wave will propagate in this direction. Pancharatnam (1955a) predicted the unexpected outcome that 
the polarization will change from the initial $\boldsymbol{d}_{0}$ to the opposite handedness, which is propagated through the crystal, and which will be transmitted by the analyser. In an experiment with iolite, he confirmed his prediction that this singular axis will appear bright, and our calculated figure $9 c$, based on the local model (6.1), closely resembles his observation (fig. $16 b$ of Pancharatnam (1955b) and fig. 71a of Ramachandran \& Ramaseshan (1961)).

We can understand Pancharatnam's phenomenon analytically using the local model. Let the polarizer be of $\mathrm{R}$ type, that is, $d_{0 \mathrm{R}}=1, d_{0 \mathrm{~L}}=0$, as in figure $9 c$. Then (7.15) and (7.16) give

$$
\begin{aligned}
I= & \left|\boldsymbol{d}_{\text {final }} \cdot \boldsymbol{d}_{1}^{*}\right|^{2} \\
= & \frac{|X+\mathrm{i}(Y+A)|}{2|X-\mathrm{i}(Y-A)|} \\
& \quad \times\left[\cosh \left(l \operatorname{Im} \sqrt{X^{2}+Y^{2}-A^{2}+2 \mathrm{i} A X}\right)-\cos \left(l \operatorname{Re} \sqrt{X^{2}+Y^{2}-A^{2}+2 \mathrm{i} A X}\right)\right],
\end{aligned}
$$

where $l$ is proportional to the thickness of the crystal. The singular axis at $X=0$, $Y=+A$ is of $\mathrm{L}$ type and so is initially $(l=0)$ extinguished. Along this axis, equation (7.19) gives

$$
\left|\boldsymbol{d}_{\text {final }} \cdot \boldsymbol{d}_{1}^{*}\right|^{2} \underset{X \rightarrow 0, Y \rightarrow A}{\longrightarrow} A^{2} l^{2} .
$$

This increases with $l$, confirming that eventually this singular axis is bright. (The paradox of the intensity apparently increasing without bound is eliminated by reinstating the average decay $\operatorname{Im} Q$ that was neglected in the local model (6.1).)

The other singular axis, at $X=0, Y=-A$, is of $\mathrm{R}$ type and so passed by the crystal but then extinguished by the (L type) analyser. This is confirmed by a corresponding analysis of (7.19), giving

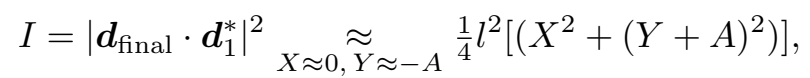

indicating that this singular axis is dark, with the intensity rising quadratically away from the zero.

For a crystal that is both dichroic and chiral, and with the polarizer/analyser arrangement, destructive interference occurs when the cosh-cos factor in (7.15) vanishes and the 'brush' prefactor does not diverge, that is, as dark spots where $\operatorname{Re}(\Delta \sigma)=2 n \pi(n=1,2, \ldots)$ on the lines $\operatorname{Im}(\Delta \sigma)=0$. These lines end on singular axes, which can therefore be located near the ends of strings of interference zeros, independently of whether the polarizer/analyser is linear, circular or elliptic. The singular axis itself is usually not a zero of $I$, because the denominator in the prefactor in (7.15) also vanishes, since the left and right eigenvectors are orthogonal at a degeneracy.

In the local model, the interference zeros are at

$$
\begin{aligned}
& X=0 \text {, } \\
& Y_{n}= \pm \sqrt{A^{2}-G^{2}+4(n \pi / l)^{2}} \quad(n=1,2, \ldots) \text {, } \\
& \left.\approx \pm \sqrt{A^{2}-G^{2}}\left(1+\frac{2 \pi^{2} n^{2}}{l^{2}\left(A^{2}-G^{2}\right)}\right) \quad(n \gg 1), \quad\right\}
\end{aligned}
$$

Proc. R. Soc. Lond. A (2003) 


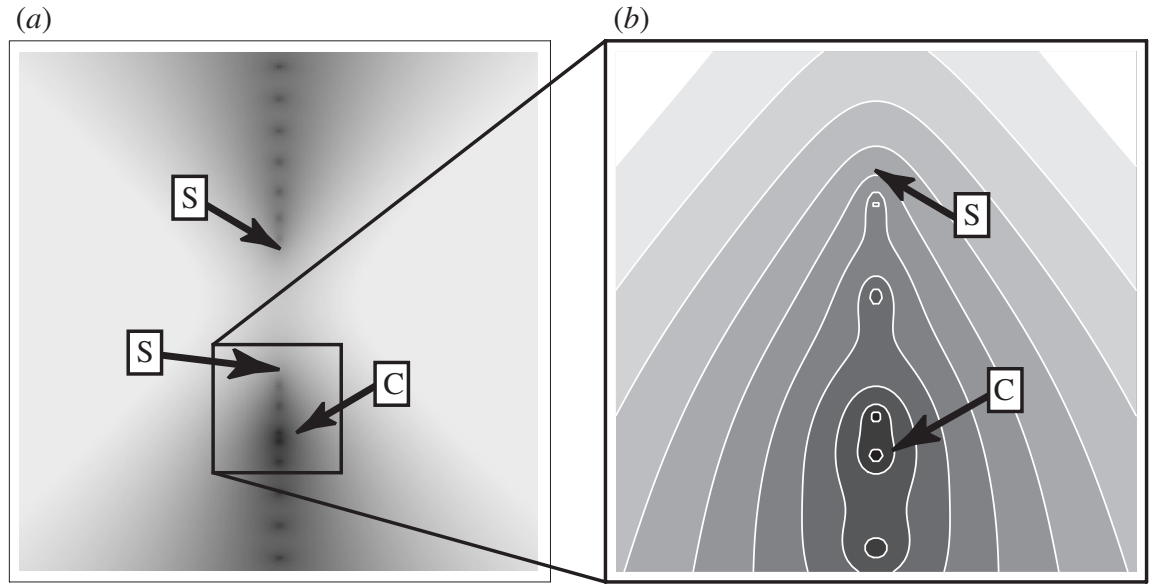

(c)

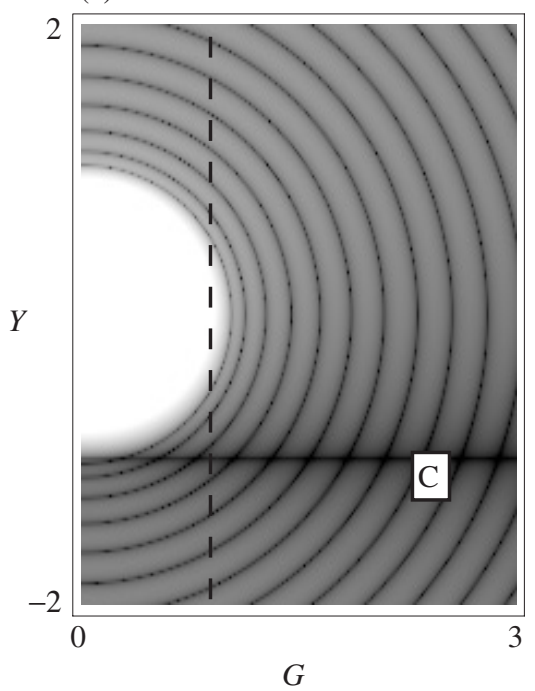

$(d)$

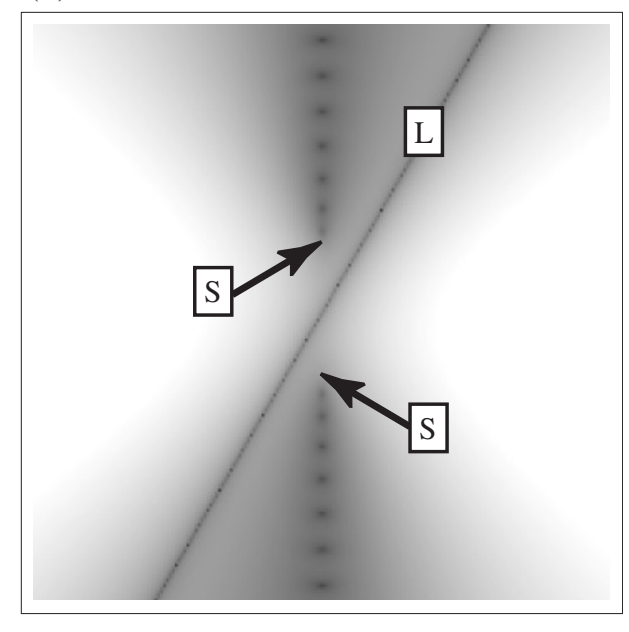

Figure 10. Density plots of $\log I$ for dichroic chiral crystals between crossed polarizer and analyser. (a) Crystal with $A=1, G=\frac{1}{2} \sqrt{3}, l=24$, right-handed polarizer and left-handed analyser, for $|X|<2,|Y|<2$; destructive interference gives two lines of dark spots, ending near the singular axes (labelled 'S') at $X=0, Y= \pm \frac{1}{2}$ (equation (6.4)); the additional dark spot at $Y=-1$ is the extinguished $\mathrm{C}$ point (equation (6.5)) (labelled ' $\mathrm{C}$ '). (b) Magnification of $(a)$, including contours of $I$, showing the $\mathrm{C}$ point, and the location of the singular axis, more clearly. $(c) \log I$ for $X=0$ as a function of $Y$ and $G$, with $A=1, l=24$, right-handed polarizer and left-handed analyser; the straight black line is the locus of the extinguished $\mathrm{C}$ point, which, according to the haunting theorem, remains fixed as $G$ increases and the singular axes, whose locus (6.4) is the semicircle, migrates away from it; the square-root branch points at the degeneracies are visible as the crowding of the interference fringes near the semicircle; the dashed line is the $G$ values corresponding to $(a),(b)$ and $(d)$; between the bright centre $Y=G=0$ and the region striated with interference fringes, $I$ falls by a factor of order $10^{9} .(d)$ As $(a)$, with linear polarizer at $\gamma=\frac{1}{6} \pi$, and crossed analyser; the L line (equation (6.6)) (in this case an isogyre) is the diagonal black line, and the $\mathrm{C}$ point is not visible. 
so, for an optically thick crystal, the spacing of the zeros increases away from the singular axis and then tends to a constant value. This is a consequence of the general phenomenon that the singular axes are square-root branch points, unlike the optic axes where difference of refractive indices vanishes linearly. Figure $10 a$ shows the two strings of zeros, visible as dark spots emanating from the vicinity of the singular axes.

When the polarizer and analyser are circular, the $\mathrm{C}$ points also appear as dark spots: for a right-handed polarizer, the right-handed $\mathrm{C}$ points are dark, and vice versa (this is a special case of the lack of symmetry between polarizer and analyser described at the end of the previous section). Figure $10 b$ (a magnification of figure $10 a$ ) shows one of these additional zeros, hiding among the interference zeros on the line emanating from the singular axis. A plot of positions of zeros on the line, as chirality increases (figure 10c), shows very clearly how each $\mathrm{C}$ point remains fixed, according to the haunting theorem, as a singular axis migrates away from it, and how the $\mathrm{C}$ point is repeatedly crossed by interference zeros.

The L lines can be revealed with crossed linear polarizer and analyser. Suppose that the orientation $\gamma$ of the polarizer coincides with the direction of a polarization on the L line $\left(\boldsymbol{d}^{+}\right.$, say); then, of course, that component of the resolved wave (7.3) is passed by the polarizer. Less obviously, the strength of the other wave $\boldsymbol{d}^{-}$(which will generally not be linearly polarized) vanishes; this follows from (7.3) and the biorthogonality relation (A 2). Therefore, only the wave $\boldsymbol{d}^{+}$survives, and it is extinguished by the analyser. This implies that the $\mathrm{L}$ lines are the loci of moving dark spots as the polarizer/analyser combination is rotated (changing $\gamma$ ) with the crystal held fixed. (This method of detecting $L$ lines for the separate polarizations $\boldsymbol{d}^{+}$and $\boldsymbol{d}^{-}$resembles that used by Angelsky et al. (2002) for optical fields with a single state of polarization.)

Along an L line, it often happens that the polarization is almost constant; then the dark spot moves very rapidly as $\gamma$ is varied. An extreme example occurs as a special feature of the local model: the L lines (figure $8 c$ ) are also isogyres, so that each of the segments of the two $\mathrm{V}$ shapes with the same direction can be revealed with a single value of $\gamma$. Calculation shows these values to be (cf. (6.6)) $\gamma=\frac{1}{2} \arccos (G / A)$ and $\gamma=\frac{1}{2}[\pi-\arccos (G / A)]$ (the result holds also for circular dichroism, that is, $G$ complex, if $G$ in these relations is replaced by $\operatorname{Re} G)$. Figure $10 d$ illustrates this phenomenon.

In general, however, a particular polarizer orientation $\gamma$ will select only part of the L line. This is illustrated in parts $(a)$ and $(b)$ of figure 11, for the crystal whose $\mathrm{L}$ line is shown magnified in figure $5 h$. Figure $11 c$ shows how this selection does not occur if the linear polarizer is replaced by a circular one; instead, a $\mathrm{C}$ point appears among the interference spots, as discussed previously.

Some of the effects described in this section are extremely delicate. They require thick crystals (so that several interference zeros separate a singular axis from a C point); consequently, absorption will greatly diminish the intensity emerging from the crystal. The observation and systematic exploration of these effects is a challenge for experimental optics.

M.V.B.'s research is supported by The Royal Society. M.R.D.'s research is supported by the Leverhulme Trust.

Proc. R. Soc. Lond. A (2003) 
(a)

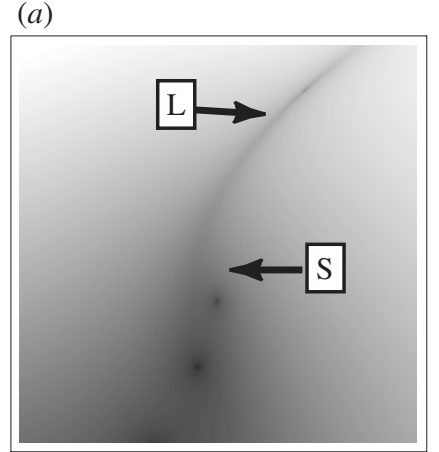

(b)

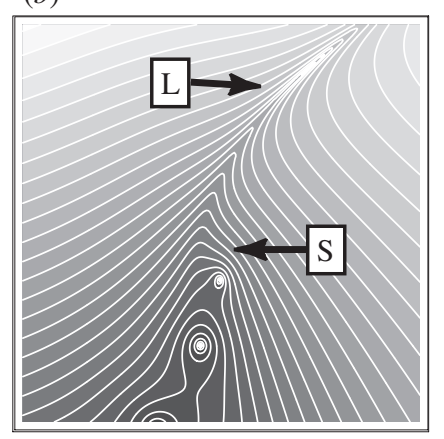

$(c)$

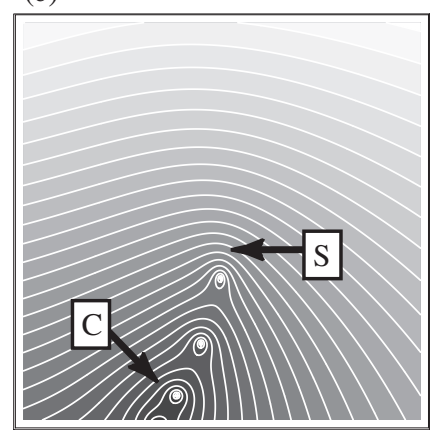

Figure 11. $\log I$ for crystal and scale as in figure $5 h$, and $k L=3500$. (a) Linear polarizer at $\gamma=1.32 \pi$, crossed analyser; the curved dark line is part of the L line, and the line of dark dots (two are shown here) ends near the singular axis $S$. (b) as $(a)$, with white contours of $I$ to make the features more clearly visible. $(c)$ as $(b)$, but with crossed circular polarizer and analyser; there is no trace of the $\mathrm{L}$ line, but a $\mathrm{C}$ point (indicated) appears as an additional dark spot encircled by white contour loops.

\section{Appendix A. Biorthogonality and other relations}

The polarizations $\boldsymbol{d}$ are the right eigenvectors of matrices $\boldsymbol{m}$, acting from the left as in (3.7). Now we need the left eigenvectors $\overline{\boldsymbol{d}}$, defined equivalently in terms of row vectors $\overline{\boldsymbol{d}}^{\mathrm{T}}$ operated on by $\boldsymbol{m}$ from the right, or column vectors $\overline{\boldsymbol{d}}$ operated on by the transpose matrix $\boldsymbol{m}^{\mathrm{T}}$ from the left. Thus the left eigenvectors are defined by

$$
\overline{\boldsymbol{d}}^{ \pm}(\boldsymbol{R})^{\mathrm{T}} \cdot \boldsymbol{m}(\boldsymbol{R}) \cdot=\lambda^{ \pm}(\boldsymbol{R}) \overline{\boldsymbol{d}}^{ \pm}(\boldsymbol{R})^{\mathrm{T}}, \quad \text { i.e. } \boldsymbol{m}^{\mathrm{T}}(\boldsymbol{R}) \cdot \overline{\boldsymbol{d}}^{ \pm}(\boldsymbol{R})=\lambda^{ \pm}(\boldsymbol{R}) \overline{\boldsymbol{d}}^{ \pm}(\boldsymbol{R}) .
$$

All relations to follow will be stated without proof; their derivations are elementary.

The left and right eigenvectors form a biorthogonal set, that is,

$$
\overline{\boldsymbol{d}}^{ \pm} \cdot \boldsymbol{d}^{\mp}=0 .
$$

(For the familiar case where $\boldsymbol{m}$ is Hermitian, that is, $\boldsymbol{m}^{T}=\boldsymbol{m}^{*}$, so $\overline{\boldsymbol{d}}^{ \pm}=\boldsymbol{d}^{ \pm *}$, and (A 2) becomes the orthogonality relation based on the complex scalar product.)

Biorthogonality enables the left eigenvectors to be written in terms of the right eigenvectors (using an arbitrary basis where the components are labelled by subscripts ' 1 ', '2') as follows:

$$
\boldsymbol{d}^{ \pm}=\left(\begin{array}{c}
d_{1}^{ \pm} \\
d_{2}^{ \pm}
\end{array}\right) \quad \Rightarrow \quad \overline{\boldsymbol{d}}^{ \pm}=\left(\begin{array}{c}
-d_{2}^{\mp} \\
d_{1}^{\mp}
\end{array}\right) .
$$

Biorthogonality also implies the completeness relation (resolution of the unit dyadic)

$$
\frac{\boldsymbol{d}^{+}\left(\overline{\boldsymbol{d}}^{+}\right)^{\mathrm{T}}}{\overline{\boldsymbol{d}}^{+} \cdot \boldsymbol{d}^{+}}+\frac{\boldsymbol{d}^{-}\left(\overline{\boldsymbol{d}}^{-}\right)^{\mathrm{T}}}{\overline{\boldsymbol{d}}^{-} \cdot \boldsymbol{d}^{-}}=\boldsymbol{I} .
$$

The scalar product of each right eigenvector with its corresponding left eigenvector is not unity (as is implied by the normalization (3.11) in the Hermitian case). However, these two scalar products are related by

$$
\bar{d}^{+} \cdot d^{+}=-\bar{d}^{-} \cdot d^{-} .
$$

Proc. R. Soc. Lond. A (2003) 
The scalar product of each right/left eigenvector with the complex conjugate of the opposite right/left eigenvector is not zero (as it would be in the Hermitian case). However, these two scalar products are related by

$$
\boldsymbol{d}^{+*} \cdot \boldsymbol{d}^{-}=\overline{\boldsymbol{d}}^{-*} \cdot \overline{\boldsymbol{d}}^{+} .
$$

The two different types of scalar product are related by

$$
\left|\overline{\boldsymbol{d}}^{+} \cdot \boldsymbol{d}^{+}\right|^{2}+\left|\boldsymbol{d}^{+*} \cdot \boldsymbol{d}^{-}\right|^{2}=1 .
$$

Transformation to a different basis, induced by a unitary operator $\boldsymbol{U}$, that is,

$$
\boldsymbol{a}^{\prime}=\boldsymbol{U} \boldsymbol{a},
$$

conserves the complex scalar product,

$$
\boldsymbol{a}^{\prime *} \cdot \boldsymbol{b}^{\prime}=\boldsymbol{a}^{*} \cdot \boldsymbol{b}
$$

Less obvious is the transformation of the non-complex scalar product of a left eigenvector with any vector, namely,

$$
\overline{\left(\boldsymbol{a}^{\prime \pm}\right)} \cdot \boldsymbol{b}^{\prime}=\operatorname{det} \boldsymbol{U} \overline{\boldsymbol{a}}^{ \pm} \cdot \boldsymbol{b}=\exp (\mathrm{i} \mu) \overline{\boldsymbol{a}}^{ \pm} \cdot \boldsymbol{b},
$$

where $\mu$ is the sum of eigenphases of $\boldsymbol{U}$. Since $|\operatorname{det} \boldsymbol{U}|=1$,

$$
\left|\overline{\left(\boldsymbol{a}^{\prime \pm}\right)} \cdot \boldsymbol{b}^{\prime}\right|=\left|\overline{\boldsymbol{a}}^{ \pm} \cdot \boldsymbol{b}\right| \text {. }
$$

\section{References}

Angelsky, O. V., Mokhun, I. I., Mokhun, A. I. \& Soskin, M. S. 2002 Interferometric methods in diagnostics of polarization singularities. Phys. Rev. E 65, 036602.

Azzam, R. M. A. \& Bashara, N. M. 1977 Ellipsometry and polarized light. Amsterdam: NorthHolland.

Berry, M. V. 1994 Pancharatnam, virtuoso of the Poincaré sphere: an appreciation. Curr. Sci. 67, 220-223.

Berry, M. V. 1998 Much ado about nothing: optical dislocation lines (phase singularities, zeros, vortices, ... ). In Singular optics, vol. 3487, pp. 1-5 (ed. M. S. Soskin). Chicago, IL: SPIE.

Berry, M. V. 2001 Geometry of phase and polarization singularities, illustrated by edge diffraction and the tides. In Singular optics 2000, vol. 4403, pp. 1-12 (ed. M. S. Soskin \& M. V. Vasnetsov). Chicago, IL: SPIE.

Berry, M. V. 2003 Mode degeneracies and the Petermann excess-noise factor for unstable lasers. J. Mod. Opt. 50, 63-81.

Berry, M. V. \& Dennis, M. R. 2001 Polarization singularities in isotropic random vector waves. Proc. R. Soc. Lond. A 457, 141-155.

Berry, M. V. \& Klein, S. 1996 Geometric phases from stacks of crystal plates. J. Mod. Opt. 43, 165-180.

Berry, M. V. \& Upstill, C. 1980 Catastrophe optics: morphologies of caustics and their diffraction patterns. Prog. Opt. 18, 257-346.

Berry, M. V. \& Wilkinson, M. 1984 Diabolical points in the spectra of triangles. Proc. R. Soc. Lond. A 392, 15-43.

Berry, M. V., Bhandari, R. \& Klein, S. 1999 Black plastic sandwiches demonstrating biaxial optical anisotropy. Eur. J. Phys. 20, 1-14.

Born, M. 1933 Optik: ein Lehrbuch der Elektromagnetischen Lichttheorie. Springer.

Proc. R. Soc. Lond. A (2003) 
Born, M. \& Wolf, E. 1959 Principles of optics. Oxford: Pergamon.

Boulanger, P. \& Hayes, M. 1990 Electromagnetic plane waves in anisotropic media: an approach using bivectors. Phil. Trans. R. Soc. Lond. A 330, 335-393.

Brosseau, C. 1998 Fundamentals of polarised light: a statistical optics approach. Wiley.

Dennis, M. R. 2002 Polarization singularities in paraxial vector fields: morphology and statistics. Opt. Commun. 213, 201-221.

Freund, I. 2002 Second harmonic generation of optical ellipse fields. Opt. Commun. 213, 129149.

Freund, I., Soskin, M. \& Mokhun, A. I. 2002 Elliptic critical points in paraxial optical fields. Opt. Commun. 208, 223-253.

Gibbs, J. W. 1928 Vector analysis. In Collected works, part 2, vol. II, pp. 17-91. New York: Longman.

Hayes, M. 1984 Inhomogeneous plane waves. Arch. Ration. Mech. Analysis 85, 41-79.

Heiss, W. D. 2000 Repulsion of resonance states and exceptional points. Phys. Rev. E 61, 929932.

Heiss, W. D. \& Harney, H. L. 2001 The chirality of exceptional points. Eur. Phys. J. D 17, 149-151.

Keck, F., Korsch, H. J. \& Mossman, S. 2003 Unfolding a diabolic point: a generalized crossing scenario. J. Phys. A 36, 2125-2137.

Korsch, H. J. \& Mossman, S. 2003 Stark resonances for a double $\delta$ quantum well: crossing scenarios, exceptional points and geometric phases. J. Phys. A 36, 2139-2153.

Landau, L. D., Lifshitz, E. M. \& Pitaevskii, L. P. 1984 Electrodynamics of continuous media. Oxford: Pergamon.

Nye, J. F. $1983 a$ Lines of circular polarization in electromagnetic wave fields. Proc. R. Soc. Lond. A 389, 279-290.

Nye, J. F. $1983 b$ Polarization effects in the diffraction of electromagnetic waves: the role of disclinations. Proc. R. Soc. Lond. A 387, 105-132.

Nye, J. F. 1995 Physical properties of crystals, 2nd edn. Oxford: Clarendon.

Nye, J. F. 1999 Natural focusing and fine structure of light: caustics and wave dislocations. Bristol: Institute of Physics.

Nye, J. F. \& Berry, M. V. 1974 Dislocations in wave trains. Proc. R. Soc. Lond. A 336, 165-190.

Pancharatnam, S. $1955 a$ The propagation of light in absorbing biaxial crystals. I. Theoretical. Proc. Ind. Natl Sci. Acad. 42, 86-109.

Pancharatnam, S. $1955 b$ The propagation of light in absorbing biaxial crystals. II. Experimental. Proc. Ind. Natl Sci. Acad. 42, 235-248.

Poincaré, H. 1892 Théorie Mathématique de la Lumière, vol. II. L'Association Amicale des Élèves et Anciens Élèves de la Faculté des Sciences. (Reprinted 1995 Paris: Éditions Jacques Gabay.)

Ramachandran, G. N. \& Ramaseshan, S. 1961 Crystal optics. In Handbuch der Physik, vol. XXV/I (ed. H. Flügge). Springer.

Rotter, I. 2001 Correlations in quantum systems and branch points in the complex plane. Phys. Rev. C 64, 034301.

Rotter, I. 2002 Branch points in the complex plane and geometric phases. Phys. Rev. E65, 026217.

Series, G. W. (ed.) 1975 Collected works of S. Pancharatnam. Oxford University Press.

Synge, J. L. 1964 The Petrov classification of gravitational fields. Commun. Dubl. Inst. Adv. Stud. A 15, 1-51.

Vasnetsov, M. \& Staliunas, K. (eds) 1999 Optical vortices. New York: Nova Science.

As this paper exceeds the maximum length normally permitted, the authors have agreed to contribute to production costs.

Proc. R. Soc. Lond. A (2003) 